\title{
Zeros of Airy Function and Relaxation Process
}

\author{
Makoto Katori *and Hideki Tanemura ${ }^{\dagger}$
}

29 September 2009

\begin{abstract}
One-dimensional system of Brownian motions called Dyson's model is the particle system with long-range repulsive forces acting between any pair of particles, where the strength of force is $\beta / 2$ times the inverse of particle distance. When $\beta=2$, it is realized as the Brownian motions in one dimension conditioned never to collide with each other. For any initial configuration, it is proved that Dyson's model with $\beta=2$ and $N$ particles, $\boldsymbol{X}(t)=\left(X_{1}(t), \ldots, X_{N}(t)\right), t \in[0, \infty), 2 \leq N<\infty$, is determinantal in the sense that any multitime correlation function is given by a determinant with a continuous kernel. The Airy function $\operatorname{Ai}(z)$ is an entire function with zeros all located on the negative part of the real axis $\mathbb{R}$. We consider Dyson's model with $\beta=2$ starting from the first $N$ zeros of $\operatorname{Ai}(z)$, $0>a_{1}>\cdots>a_{N}, N \geq 2$. In order to properly control the effect of such initial confinement of particles in the negative region of $\mathbb{R}$, we put the drift term to each Brownian motion, which increases in time as a parabolic function : $Y_{j}(t)=X_{j}(t)+t^{2} / 4+\left\{d_{1}+\sum_{\ell=1}^{N}\left(1 / a_{\ell}\right)\right\} t, 1 \leq j \leq N$, where $d_{1}=\operatorname{Ai}^{\prime}(0) / \operatorname{Ai}(0)$. We show that, as the $N \rightarrow \infty$ limit of $\boldsymbol{Y}(t)=\left(Y_{1}(t), \ldots, Y_{N}(t)\right), t \in[0, \infty)$, we obtain an infinite particle system, which is the relaxation process from the configuration, in which every zero of $\operatorname{Ai}(z)$ on the negative $\mathbb{R}$ is occupied by one particle, to the stationary state $\mu_{\mathrm{Ai}}$. The stationary state $\mu_{\mathrm{Ai}}$ is the determinantal point process with the Airy kernel, which is spatially inhomogeneous on $\mathbb{R}$ and in which the Tracy-Widom distribution describes the rightmost particle position.
\end{abstract}

KEY WORDS: Zeros of Airy function; Relaxation process; Dyson's model; Determinantal point process; Entire function; Weierstrass canonical product

${ }^{*}$ Department of Physics, Faculty of Science and Engineering, Chuo University, Kasuga, Bunkyoku, Tokyo 112-8551, Japan; e-mail: katori@phys.chuo-u.ac.jp

${ }^{\dagger}$ Department of Mathematics and Informatics, Faculty of Science, Chiba University, 1-33 Yayoicho, Inage-ku, Chiba 263-8522, Japan; e-mail: tanemura@math.s.chiba-u.ac.jp 


\section{Introduction}

\subsection{Dyson's model: One-Dimensional Brownian Particle Sys- tem Interacting through Pair Force $1 / x$}

To understand the time-evolution of distributions of interacting particle systems on a large space-time scale (thermodynamic and hydrodynamic limits) is one of the main topics of statistical physics. If the interactions among particles are short ranged, the standard theory is useful. If they are long ranged, however, general theory has not yet been established and thus detailed study of model systems is required [26].

In the present paper, we consider Brownian particles in one dimension with longranged repulsive forces acting between any pair of particles, where the strength of force is exactly equal to $1 / x$ when the particle distance is $x$. If the number of particles is finite $N<\infty$, the system is described by $\Xi(t)=\sum_{j=1}^{N} \delta_{X_{j}(t)}, 2 \leq N<\infty$, where $\boldsymbol{X}(t)=\left(X_{1}(t), \ldots, X_{N}(t)\right)$ satisfies the following system of stochastic differential equations (SDEs);

$$
d X_{j}(t)=d B_{j}(t)+\sum_{\substack{1 \leq k \leq N \\ k \neq j}} \frac{1}{X_{j}(t)-X_{k}(t)} d t, \quad 1 \leq j \leq N, \quad t \in[0, \infty)
$$

with independent one-dimensional standard Brownian motions $B_{j}(t), 1 \leq j \leq N$. The SDEs obtained by replacing the $1 / x$ force in (1.1) by $\beta /(2 x)$ with a parameter $\beta>0$ were introduced by Dyson [4] to understand the statistics of eigenvalues of hermitian random matrices as particle distributions of interacting Brownian motions in $\mathbb{R}$. Corresponding to the special values $\beta=1,2$ and 4 , hermitian random matrices are in the three statistical ensembles with different symmetries, called the Gaussian orthogonal ensemble (GOE), the Gaussian unitary ensemble (GUE), and the Gaussian symplectic ensemble (GSE), respectively [5]. In particular for $\beta=2$, that is the case of (1.1), if the eigenvalue distribution of $N \times N$ hermitian random matrices in the GUE with variance $\sigma^{2}$ is denoted by $\mu_{N, \sigma^{2}}^{\mathrm{GUE}}$, we can show

$$
\lim _{N \rightarrow \infty} \mu_{N, 2 N / \pi^{2}}^{\mathrm{GUE}}(\cdot)=\mu_{\sin }(\cdot)
$$

where $\mu_{\text {sin }}$ denotes the determinantal (Fermion) point process [24, 23] with the socalled sine kernel

$$
K_{\sin }(x)=\frac{1}{2 \pi} \int_{|k| \leq \pi} d k e^{\sqrt{-1} k x}=\frac{\sin (\pi x)}{\pi x}, \quad x \in \mathbb{R} .
$$

That is, $\mu_{\text {sin }}$ is a spatially homogeneous particle distribution, in which the particle density is given by $\rho_{\sin }=\lim _{x \rightarrow 0} K_{\sin }(x)=1$ and any $N_{1}$-point correlation function $\rho_{\text {sin }}\left(\boldsymbol{x}_{N_{1}}\right), \boldsymbol{x}_{N_{1}}=\left(x_{1}, \ldots, x_{N_{1}}\right) \in \mathbb{R}^{N_{1}}, N_{1} \geq 2$, is given by a determinant of an $N_{1} \times N_{1}$ real symmetric matrix;

$$
\rho_{\sin }\left(\boldsymbol{x}_{N_{1}}\right)=\operatorname{det}_{1 \leq j, k \leq N_{1}}\left[K_{\sin }\left(x_{j}-x_{k}\right)\right] .
$$


Based on this fact known in the random matrix theory [17], Spohn [25] studied the equilibrium dynamics obtained in the infinite-particle limit $N \rightarrow \infty$ of Dyson's model (1.1). Since the $1 / x$ force is not summable, in the infinite-particle limit $N \rightarrow \infty$ the sum in (1.1) should be regarded as an improper sum, in the sense that for $X_{j}(t) \in[-L, L]$ the summation is restricted to $k$ 's such that $X_{k}(t) \in[-L, L]$ and then the limit $L \rightarrow \infty$ is taken. It is expected that the dynamics with an infinite number of particles can exist only for initial configurations having the same asymptotic density to the right and left [25, 13].

The problem, which we address in the present paper, is how we can control Dyson's model with an infinite number of particles starting from asymmetric initial configurations. The motivation is again coming from the random matrix theory as follows. Consider the Airy function [1, 30]

$$
\operatorname{Ai}(z)=\frac{1}{2 \pi} \int_{\mathbb{R}} d k e^{\sqrt{-1}\left(z k+k^{3} / 3\right)}
$$

It is a solution of Airy's equation $f^{\prime \prime}(z)-z f(z)=0$ with the asymptotics on the real axis $\mathbb{R}$ :

$$
\begin{aligned}
& \operatorname{Ai}(x) \simeq \frac{1}{2 \sqrt{\pi} x^{1 / 4}} \exp \left(-\frac{2}{3} x^{3 / 2}\right) \\
& \operatorname{Ai}(-x) \simeq \frac{1}{\sqrt{\pi} x^{1 / 4}} \cos \left(\frac{2}{3} x^{3 / 2}-\frac{\pi}{4}\right) \quad \text { in } \quad x \rightarrow+\infty
\end{aligned}
$$

In the GUE random matrix theory, the following scaling limit has been extensively studied:

$$
\lim _{N \rightarrow \infty} \mu_{N, N^{1 / 3}}^{\mathrm{GUE}}\left(2 N^{2 / 3}+\cdot\right)=\mu_{\mathrm{Ai}}(\cdot)
$$

where $\mu_{\mathrm{Ai}}$ is the determinantal point process such that the correlation kernel is given by [8, 28],

$$
\begin{aligned}
K_{\mathrm{Ai}}(y \mid x) & =\int_{0}^{\infty} d u \operatorname{Ai}(u+x) \operatorname{Ai}(u+y) \\
& = \begin{cases}\frac{\operatorname{Ai}(x) \operatorname{Ai}^{\prime}(y)-\operatorname{Ai}^{\prime}(x) \operatorname{Ai}(y)}{x-y}, & x \neq y \in \mathbb{R} \\
\left(\operatorname{Ai}^{\prime}(x)\right)^{2}-x(\operatorname{Ai}(x))^{2}, & x=y \in \mathbb{R} .\end{cases}
\end{aligned}
$$

It is another infinite-particle limit different from (1.2) and is called the soft-edge scaling limit, since $x^{2} / 2 t \simeq\left(2 N^{2 / 3}\right)^{2} /\left(2 N^{1 / 3}\right)=2 N$ marks the right edge of semicircleshaped profile of the GUE eigenvalue distribution (see, for example, [12]). The particle distribution $\mu_{\mathrm{Ai}}$ with the Airy kernel (1.7) is highly asymmetric: As a matter of fact, the particle density $\rho_{\mathrm{Ai}}(x)=K_{\mathrm{Ai}}(x \mid x)$ decays rapidly to zero as $x \rightarrow \infty$, but it diverges

$$
\rho_{\mathrm{Ai}}(x) \simeq \frac{1}{\pi}(-x)^{1 / 2} \rightarrow \infty \quad \text { as } \quad x \rightarrow-\infty
$$


Let $R$ be the position of the rightmost particle on $\mathbb{R}$ in $\mu_{\mathrm{Ai}}$. Then its distribution is given by the celebrated Tracy-Widom distribution [28]

$$
\mu_{\mathrm{Ai}}(R<x)=\exp \left[-\int_{x}^{\infty}(y-x)(q(y))^{2} d y\right],
$$

where $q(x)$ is the unique solution of the Painlevé II equation

$$
q^{\prime \prime}=x q+2 q^{3}
$$

satisfying the boundary condition $q(x) \simeq \operatorname{Ai}(x)$ in $x \rightarrow \infty$. Prähofer and Spohn $[22$ and Johansson [11] studied the equilibrium fluctuation of this rightmost particle and called it the Airy process. Tracy and Widom derived a system of partial differential equations, which govern the Airy process [29]. See also [2, 3]. How can we realize $\mu_{\mathrm{Ai}}$ as the equilibrium state of Brownian infinite-particle system interacting through pair force $1 / x$ ? The initial configurations should be asymmetric, but what kinds of conditions should be satisfied by them ? How should we modify the SDEs of original Dyson's model (1.1), when we provide finite-particle approximations for such asymmetric infinite particle systems?

In the present paper, as an explicit answer to the above questions, we will present a relaxation process with an infinite number of particles converging to the stationary state $\mu_{\mathrm{Ai}}$ in $t \rightarrow \infty$. Its initial configuration is given by

$$
\xi_{\mathcal{A}}(\cdot)=\sum_{a \in \mathcal{A}} \delta_{a}(\cdot)=\sum_{j=1}^{\infty} \delta_{a_{j}}(\cdot)
$$

in which every zero of the Airy function (1.4) is occupied by one particle. This special choice of the initial configuration is due to the fact that the zeros of the Airy function are located only on the negative part of the real axis $\mathbb{R}$,

$$
\mathcal{A} \equiv \mathrm{Ai}^{-1}(0)=\left\{a_{j}, j \in \mathbb{N}: \operatorname{Ai}\left(a_{j}\right)=0,0>a_{1}>a_{2}>\cdots\right\}
$$

with the values [1] $a_{1}=-2.33 \ldots, a_{2}=-4.08 \ldots, a_{3}=-5.52 \ldots, a_{4}=-6.78 \ldots$, and that they admit the asymptotics [1, 30]

$$
a_{j} \simeq-\left(\frac{3 \pi}{2}\right)^{2 / 3} j^{2 / 3} \quad \text { in } \quad j \rightarrow \infty .
$$

Then the average density of zeros of the Airy function around $x$, denoted by $\rho_{\mathrm{Ai}^{-1}(0)}(x)$, behaves as

$$
\rho_{\mathrm{Ai}^{-1}(0)}(x) \simeq \frac{1}{\pi}(-x)^{1 / 2} \rightarrow \infty \quad \text { as } \quad x \rightarrow-\infty,
$$

which coincides with (1.8). The approximation of our process with a finite number of particles $N<\infty$ is given by $\Xi_{\mathcal{A}}(t)=\sum_{j=1}^{N} \delta_{Y_{j}(t)}$ with

$$
Y_{j}(t)=X_{j}(t)+\frac{t^{2}}{4}+D_{\mathcal{A}_{N}} t, \quad 1 \leq j \leq N, \quad t \in[0, \infty)
$$


associated with the solution $\boldsymbol{X}(t)=\left(X_{1}(t), \ldots, X_{N}(t)\right)$ of Dyson's model (1.1), where

$$
D_{\mathcal{A}_{N}}=d_{1}+\sum_{\ell=1}^{N} \frac{1}{a_{\ell}} \text {. }
$$

Here $d_{1}=\operatorname{Ai}^{\prime}(0) / \operatorname{Ai}(0)$ and $\mathcal{A}_{N} \equiv\left\{0>a_{1}>\cdots>a_{N}\right\} \subset \mathcal{A}$ is the sequence of the first $N$ zeros of the Airy function. In other words, $\boldsymbol{Y}(t)=\left(Y_{1}(t), Y_{2}(t), \ldots, Y_{N}(t)\right)$ satisfies the following SDEs ;

$$
\begin{aligned}
& d Y_{j}(t)=d B_{j}(t)+\left(\frac{t}{2}+D_{\mathcal{A}_{N}}\right) d t+\sum_{\substack{1 \leq k \leq N \\
k \neq j}} \frac{d t}{Y_{j}(t)-Y_{k}(t)} \\
&=d B_{j}(t)+\sum_{\substack{1 \leq k \leq N \\
k \neq j}}\left(\frac{1}{Y_{j}(t)-Y_{k}(t)}+\frac{1}{a_{k}}\right) d t+\left(\frac{t}{2}+d_{1}+\frac{1}{a_{j}}\right) d t, \\
& 1 \leq j \leq N, \quad t \in[0, \infty)
\end{aligned}
$$

where $B_{j}(t)$ 's are independent one-dimensional standard Brownian motions.

For $\boldsymbol{Y}(0)=\boldsymbol{x} \in \mathbb{R}^{N}$, set $\xi^{N}(\cdot)=\sum_{j=1}^{N} \delta_{x_{j}}(\cdot)$ and consider the process $\Xi_{\mathcal{A}}(t)$ starting from the configuration $\xi^{N}$. We consider a set of initial configurations $\xi^{N}$ such that they are in general different from the $N$-particle approximation of (1.9),

$$
\xi_{\mathcal{A}}^{N}(\cdot)=\sum_{a \in \mathcal{A}_{N}} \delta_{a}(\cdot)=\sum_{j=1}^{N} \delta_{a_{j}}(\cdot),
$$

but the particle density $\rho(x)$ of $\lim _{N \rightarrow \infty} \xi^{N}$ will show the same asymptotic in $x \rightarrow$ $-\infty$ as (1.8). Because of the strong repulsive forces acting between particle pairs in (1.1), such confinement of particles in the negative region of $\mathbb{R}$ at the initial time causes strong positive drifts of Brownian particles. The coefficient (1.13) of the drift term $D_{\mathcal{A}_{N}} t$ added in (1.12), however, negatively diverges

$$
D_{\mathcal{A}_{N}} \simeq-\left(\frac{12}{\pi^{2}}\right)^{1 / 3} N^{1 / 3} \rightarrow-\infty \quad \text { as } \quad N \rightarrow \infty .
$$

We will determine a class of asymmetric initial configurations denoted by $\mathfrak{X}_{0}^{\mathcal{A}}$, which includes $\xi_{\mathcal{A}}$ as a typical one, such that the effect on dynamics of asymmetry in configuration will be compensated by the additional drift term $D_{\mathcal{A}_{N}} t$ in the infiniteparticle limit $N \rightarrow \infty$ and the dynamics with an infinite number of particles exists. Note that we should take $N \rightarrow \infty$ limit for finite $t<\infty$ in our process (1.12) to discuss non-equilibrium dynamics with an infinite number of particles. In the class $\mathfrak{X}_{0}^{\mathcal{A}}$, when the initial configuration is specially set to be $\xi_{\mathcal{A}}$, (1.9), we can prove that the dynamics shows a relaxation in the long-term limit $t \rightarrow \infty$ to the equilibrium dynamics in $\mu_{\mathrm{Ai}}$. In the proof we use the special property of the systems (1.1) 
and (1.14) such that the processes have space-time determinantal correlations. This feature comes from the fact that if and only if the strength of pair force is exactly equal to $1 / x$ when the particle distance is $x$, i.e., iff $\beta=2$, Dyson's model is realized as the Brownian motions conditioned never to collide with each other [10, 12.

In order to explain the importance of the notion of entire functions for the present problem, we rewrite the results reported in our previous paper [13] for Dyson's model with symmetric initial configurations below. Then the changes which we have to do for the systems with asymmetric initial configurations are shown. There the origin of the quadratic term $t^{2} / 4$ in (1.12) will be clarified.

\subsection{Processes with Space-time Determinantal Correlations and Entire Functions}

In an earlier paper [13], we studied a class of a non-equilibrium dynamics of Dyson's model with $\beta=2$ and an infinite number of particles. As an example in the class, we reported a relaxation process, denoted here by $\left(\Xi(t), \mathbb{P}_{\text {sin }}\right)$, which starts from a configuration

$$
\xi_{\mathbb{Z}}(\cdot)=\sum_{a \in \mathbb{Z}} \delta_{a}(\cdot),
$$

in which every point of $\mathbb{Z}$ is occupied by one particle, and converges to the stationary state $\mu_{\sin }$. This process $\left(\Xi(t), \mathbb{P}_{\sin }\right)$ is determinantal, in the sense that there is a function $\mathbb{K}_{\sin }(s, x ; t, y)$ called the correlation kernel such that it is continuous with respect to $(x, y) \in \mathbb{R}^{2}$ for any fixed $(s, t) \in[0, \infty)^{2}$, and that, for any integer $M \geq 1$, any sequence $\left(N_{m}\right)_{m=1}^{M}$ of positive integers, and any time sequence $0<t_{1}<\cdots<t_{M}<\infty$, the $\left(N_{1}, \ldots, N_{M}\right)$-multitime correlation function $\rho_{\text {sin }}\left(t_{1}, \boldsymbol{x}_{N_{1}}^{(1)} ; \ldots ; t_{M}, \boldsymbol{x}_{N_{M}}^{(M)}\right), \boldsymbol{x}_{N_{m}}^{(m)}=\left(x_{1}^{(m)}, \ldots, x_{N_{m}}^{(m)}\right) \in \mathbb{R}^{N_{m}}, 1 \leq m \leq M$, is expressed by a determinant of a $\sum_{m=1}^{M} N_{m} \times \sum_{m=1}^{M} N_{m}$ asymmetric real matrix;

$$
\rho_{\sin }\left(t_{1}, \boldsymbol{x}_{N_{1}}^{(1)} ; \ldots ; t_{M}, \boldsymbol{x}_{N_{M}}^{(M)}\right)=\operatorname{det}_{\substack{1 \leq j \leq N_{m}, 1 \leq k \leq N_{n} \\ 1 \leq m, n \leq M}}\left[\mathbb{K}_{\sin }\left(t_{m}, x_{j}^{(m)} ; t_{n}, x_{k}^{(n)}\right)\right] .
$$

The finite dimensional distributions of the process $\left(\Xi(t), \mathbb{P}_{\sin }\right)$ are determined by $\mathbb{K}_{\text {sin }}$ through (1.18). It is expected that the correlation kernel $\mathbb{K}_{\text {sin }}$ is described by using the sine function as is the correlation kernel $K_{\sin }$ of the stationary distribution $\mu_{\sin }$ given by (1.3). It is indeed true. Set

$$
f(z)=\sin (\pi z), \quad z \in \mathbb{C},
$$

and

$$
p_{\sin }(t, x)=\frac{e^{-x^{2} / 2 t}}{\sqrt{2 \pi|t|}}, \quad t \in \mathbb{R} \backslash\{0\}, \quad x \in \mathbb{C} .
$$

When $t>0, p_{\sin }(t, y-x)$ is the heat kernel: the solution of the heat equation $\partial u(t, x) / \partial t=(1 / 2) \partial^{2} u(t, x) / \partial x^{2}$ with $\lim _{t \rightarrow 0} u(t, x) d x=\delta_{y}(d x)$, and is expressed 
using (1.19) as

$$
p_{\sin }(t, y-x)=\frac{1}{2} \int_{\mathbb{R}} d u e^{-\pi^{2} u^{2} t / 2}\{f(u x) f(u y)+f(u x+1 / 2) f(u y+1 / 2)\} .
$$

For $0<s<t$, by setting (1.20), the Chapman-Kolmogorov equation

$$
\int_{\mathbb{R}} d y p_{\sin }(t-s, z-y) p_{\sin }(s, y-x)=p_{\sin }(t, z-x)
$$

can be extended to

$$
\int_{\mathbb{R}} d y p_{\sin }(-t, z-y) p_{\sin }(t-s, y-x)=p_{\sin }(-s, z-x) .
$$

Then $\mathbb{K}_{\sin }(s, x ; t, y)$ is given by

$$
\begin{aligned}
\mathbb{K}_{f}(s, x ; t, y)= & \sum_{a \in f^{-1}(0)} \int_{\sqrt{-1} \mathbb{R}} \frac{d z}{\sqrt{-1}} p_{f}(0, a ; s, x) \frac{1}{z-a} \frac{f(z)}{f^{\prime}(a)} p_{f}(t, y ; 0, z) \\
& -\mathbf{1}(s>t) p_{f}(t, y ; s, x), \quad s, t \geq 0, \quad x, y \in \mathbb{R}
\end{aligned}
$$

with setting (1.19) and $p_{f}(s, x ; t, y)=p_{\sin }(t-s, y-x)$ with (1.20), where $f^{-1}(0)$ denotes the zero set of the function $f ; f^{-1}(0)=\{z: f(z)=0\}, f^{\prime}(a)=d f(z) /\left.d z\right|_{z=a}$, and $\mathbf{1}(\omega)$ is the indicator of a condition $\omega ; \mathbf{1}(\omega)=1$ if $\omega$ is satisfied, and $\mathbf{1}(\omega)=0$ otherwise. In this paper $\int_{\sqrt{-1} \mathbb{R}} d z$. means the integral on the imaginary axis in $\mathbb{C}$ from $-\sqrt{-1} \infty$ to $\sqrt{-1} \infty$. The well-definedness of the correlation kernel $\mathbb{K}_{\text {sin }}$ and thus of the process $\left(\Xi(t), \mathbb{P}_{\text {sin }}\right)$ is guaranteed [13] by the fact that the sine function $(1.19)$ is an entire function (i.e., analytic in the whole complex plane $\mathbb{C}$ ), and the order of growth $\rho_{f}$, which is generally defined for an entire function $f$ by

$$
\rho_{f}=\limsup _{r \rightarrow \infty} \frac{\log \log M_{f}(r)}{\log r} \quad \text { for } \quad M_{f}(r)=\max _{|z|=r}|f(z)|,
$$

is one. (The type defined by $\sigma_{f}=\limsup _{r \rightarrow \infty} \log M_{f}(r) / r^{\rho_{f}}$ is equal to $\pi$ for (1.19). That is, the sine function (1.19) is an entire function of exponential type $\pi$ [16] ; $M_{f}(r) \sim e^{\pi r}$ as $r \rightarrow \infty$.) We can show that

$$
\mathbb{K}_{\sin }(t, x ; t, y) \mathbb{K}_{\sin }(t, y ; t, x) d x d y \rightarrow \xi_{\mathbb{Z}}(d x) \mathbf{1}(x=y) \quad \text { as } \quad t \rightarrow 0
$$

since $f^{-1}(0)=\sin ^{-1}(0) / \pi=\mathbb{Z}$. It implies that the initial configuration (1.17) of the relaxation process $\left(\Xi(t), \mathbb{P}_{\text {sin }}\right)$ shall be regarded as the point-mass distribution on the zero set of the sine function (1.19). Moreover, we showed in [13], by noting

$$
\frac{1}{z-a} \frac{f(z)}{f^{\prime}(a)}=K_{\sin }(z-a)
$$


if $a \in f^{-1}(0)=\mathbb{Z}$ and $z \neq a$ for (1.19) , that

$$
\mathbb{K}_{\sin }(s+\theta, x ; t+\theta, y) \rightarrow \mathbf{K}_{\sin }(t-s, y-x) \quad \text { as } \quad \theta \rightarrow \infty
$$

with the so-called extended sine kernel,

$$
\mathbf{K}_{\sin }(t, x)= \begin{cases}\int_{0}^{1} d u e^{\pi^{2} u^{2} t / 2} \cos (\pi u x) & \text { if } t>0 \\ K_{\sin }(x) & \text { if } t=0 \\ -\int_{1}^{\infty} d u e^{\pi^{2} u^{2} t / 2} \cos (\pi u x) & \text { if } t<0\end{cases}
$$

$x \in \mathbb{R}$. The equilibrium dynamics in $\mu_{\text {sin }}$, first studied by Spohn [25], has been shown to be determinantal with the correlation kernel (1.25) by Nagao and Forrester [18]. This process is realized in the long-term limit of the relaxation process $\left(\Xi(t), \mathbb{P}_{\sin }\right)$. See also the Dirichlet form approach by Osada to the reversible process with respect to $\mu_{\text {sin }}[20,21]$.

Now we set

$$
f(z)=\operatorname{Ai}(z), \quad z \in \mathbb{C} .
$$

The Airy function $\operatorname{Ai}(z)$, (1.4), is another entire function, whose order of growth is $\rho_{f}=3 / 2$ with type $\sigma_{f}=2 / 3 ; \max _{|z|=r}|f(z)| \sim \exp \left[(2 / 3) r^{3 / 2}\right]$ as $r \rightarrow \infty$. For $t>0$, we consider

$$
p_{\mathrm{Ai}}(t, y \mid x)=\int_{\mathbb{R}} d u e^{u t / 2} f(u+x) f(u+y), \quad x, y \in \mathbb{R},
$$

which is the solution of the differential equation;

$$
\frac{\partial}{\partial t} u(t, x)=\frac{1}{2}\left(\frac{\partial^{2}}{\partial x^{2}}-x\right) u(t, x) \quad \text { with } \quad \lim _{t \rightarrow 0} u(t, x) d x=\delta_{y}(d x) .
$$

The integral $\int_{\mathbb{R}} d z p_{\text {Ai }}(t, z \mid x)$ is given by

$$
g(t, x)=\exp \left(-\frac{t x}{2}+\frac{t^{3}}{24}\right) .
$$

We find, for $s<t<0, g(s, x) p_{\mathrm{Ai}}(t-s, y \mid x) / g(t, y)$ is equal to the transition probability density of

$$
B(t)+\frac{t^{2}}{4}
$$

from $x$ at time $s$ to $y$ at time $t, x, y \in \mathbb{R}$, where $B(t), t \in[0, \infty)$ is the onedimensional standard Brownian motion. (See also [6] and references therein.) Then 
for $s, t \in \mathbb{R}, s \neq t, x, y \in \mathbb{C}$, we set

$$
\begin{aligned}
& q(s, t, y-x)=p_{\sin }\left(t-s,\left(y-\frac{t^{2}}{4}\right)-\left(x-\frac{s^{2}}{4}\right)\right) \\
& =\frac{1}{\sqrt{2 \pi|t-s|}} \exp \left[-\frac{(y-x)^{2}}{2(t-s)}+\frac{(t+s)(y-x)}{4}-\frac{(t-s)(t+s)^{2}}{32}\right]
\end{aligned}
$$

and as an extension of (1.27) we define

$$
\begin{aligned}
& p_{\mathrm{Ai}}(t-s, y \mid x)=\frac{g(t, y)}{g(s, x)} q(s, t, y-x) \\
& =\frac{1}{\sqrt{2 \pi|t-s|}} \exp \left[-\frac{(y-x)^{2}}{2(t-s)}-\frac{(t-s)(y+x)}{4}+\frac{(t-s)^{3}}{96}\right] .
\end{aligned}
$$

Corresponding to (1.21) and (1.22), we have the two sets of equalities

$$
\begin{aligned}
& \int_{\mathbb{R}} d y q(s, t, z-y) q(0, s, y-x)=q(0, t, z-x) \\
& \int_{\mathbb{R}} d y q(t, 0, z-y) q(s, t, y-x)=q(s, 0, z-x)
\end{aligned}
$$

and

$$
\begin{aligned}
& \int_{\mathbb{R}} d y p_{\mathrm{Ai}}(t-s, z \mid y) p_{\mathrm{Ai}}(s, y \mid x)=p_{\mathrm{Ai}}(t, z \mid x) \\
& \int_{\mathbb{R}} d y p_{\mathrm{Ai}}(-t, z \mid y) p_{\mathrm{Ai}}(t-s, y \mid x)=p_{\mathrm{Ai}}(-s, z \mid x)
\end{aligned}
$$

for $0<s<t$.

Let $\mathbb{K}_{\mathrm{Ai}}$ be the function given by (1.23) with setting (1.26) and $p_{f}(s, x ; t, y)=$ $p_{\mathrm{Ai}}(t-s, y \mid x)$ with (1.31). We will prove that $\mathbb{K}_{\mathrm{Ai}}$ is well-defined as a correlation kernel and it determines finite dimensional distributions of an infinite particle system through a similar formula to (1.18). We denote this system by $\left(\Xi_{\mathcal{A}}(t), \mathbb{P}_{\mathrm{Ai}}\right)$. The fact that $p_{\mathrm{Ai}}$ used in $\mathbb{K}_{\mathrm{Ai}}$ is a transform (1.31) of the transition probability density $q$ of (1.29) is the origin of the quadratic term $t^{2} / 4$ in (1.12). We can show that

$$
\mathbb{K}_{\mathrm{Ai}}(t, x ; t, y) \mathbb{K}_{\mathrm{Ai}}(t, y ; t, x) d x d y \rightarrow \xi_{\mathcal{A}}(d x) \mathbf{1}(x=y) \quad \text { as } \quad t \rightarrow 0 .
$$

By using the integral formula for (1.26)

$$
\frac{1}{z-a} \frac{f(z)}{f^{\prime}(a)}=\frac{1}{\left(\operatorname{Ai}^{\prime}(a)\right)^{2}} \int_{0}^{\infty} d u \operatorname{Ai}(u+z) \operatorname{Ai}(u+a)
$$

for $a \in \mathcal{A}, z \neq a$, and the fact that $\left\{\operatorname{Ai}(x+a) / \operatorname{Ai}^{\prime}(a), a \in \mathcal{A}\right\}$ forms a complete orthonormal basis for the space $L^{2}(0, \infty)$ of square integrable functions on the interval $(0, \infty)$ [27], we will prove that

$$
\mathbb{K}_{\mathrm{Ai}}(s+\theta, x ; t+\theta, y) \rightarrow \mathbf{K}_{\mathrm{Ai}}(t-s, y \mid x) \quad \text { as } \quad \theta \rightarrow \infty
$$


where $\mathbf{K}_{\mathrm{Ai}}$ is the so-called extended Airy kernel,

$$
\mathbf{K}_{\mathrm{Ai}}(t, y \mid x)=\left\{\begin{array}{cc}
\int_{0}^{\infty} d u e^{-u t / 2} \operatorname{Ai}(u+x) \operatorname{Ai}(u+y) & \text { if } t \geq 0 \\
-\int_{-\infty}^{0} d u e^{-u t / 2} \operatorname{Ai}(u+x) \operatorname{Ai}(u+y) & \text { if } t<0,
\end{array}\right.
$$

$x, y \in \mathbb{R}$. We denote by $\left(\Xi_{\mathcal{A}}(t), \mathbf{P}_{\mathrm{Ai}}\right)$ the infinite particle system, which is determinantal with the correlation kernel $\mathbf{K}_{\mathrm{Ai}}$ [9, 19, 15, 12. The Airy kernel (1.7) of $\mu_{\mathrm{Ai}}$ is given by $K_{\mathrm{Ai}}(y \mid x)=\mathbf{K}_{\mathrm{Ai}}(0, y \mid x)$ and thus $\left(\Xi_{\mathcal{A}}(t), \mathbf{P}_{\mathrm{Ai}}\right)$ is a reversible process with respect to $\mu_{\mathrm{Ai}}$. The process $\left(\Xi_{\mathcal{A}}(t), \mathbb{P}_{\mathrm{Ai}}\right)$, which is determinantal with the correlation kernel $\mathbb{K}_{\mathrm{Ai}}$, is a non-equilibrium infinite particle system exhibiting the relaxation phenomenon from the initial configuration $\xi_{\mathcal{A}}$ to the stationary state $\mu_{\mathrm{Ai}}$.

Then consider the finite-particle system (1.12) again. Let $\mathbb{P}_{\mathcal{A}}^{\xi^{N}}$ be the distribution of the process $\Xi_{\mathcal{A}}(t)=\sum_{j=1}^{N} \delta_{Y_{j}(t)}$ starting from a configuration $\xi^{N}$. We denote by $\mathfrak{M}$ the space of nonnegative integer-valued Radon measures on $\mathbb{R}$, which is a Polish space with the vague topology: we say $\xi_{n}$ converges to $\xi$ vaguely, if $\lim _{n \rightarrow \infty} \int_{\mathbb{R}} \varphi(x) \xi_{n}(d x)=\int_{\mathbb{R}} \varphi(x) \xi(d x)$ for any $\varphi \in \mathrm{C}_{0}(\mathbb{R})$, where $\mathrm{C}_{0}(\mathbb{R})$ is the set of all continuous real-valued functions with compact supports. Any element $\xi$ of $\mathfrak{M}$ can be represented as $\xi(\cdot)=\sum_{j \in \Lambda} \delta_{x_{j}}(\cdot)$ with an index set $\Lambda$ and a sequence of points in $\mathbb{R}, \boldsymbol{x}=\left(x_{j}\right)_{j \in \Lambda}$ satisfying $\xi(I)=\sharp\left\{x_{j}: x_{j} \in I\right\}<\infty$ for any compact subset $I \subset \mathbb{R}$. For $A \subset \mathbb{R}$, we write the restriction of $\xi$ on $A$ as $(\xi \cap A)(\cdot)=\sum_{j \in \Lambda: x_{j} \in A} \delta_{x_{j}}(\cdot)$. We put $\mathfrak{M}_{0}=\{\xi \in \mathfrak{M}: \xi(\{x\}) \leq 1$ for any $x \in \mathbb{R}\}$. We will prove that the finite particle process $\left(\Xi_{\mathcal{A}}(t), \mathbb{P}_{\mathcal{A}}^{\xi^{N}}\right)$ is determinantal for any initial configuration $\xi^{N} \in \mathfrak{M}_{0}$ and give the correlation kernel $\mathbb{K}_{\mathcal{A}}^{\xi^{N}}$ (Proposition 2.4). For $\xi \in \mathfrak{M}$ with an infinite number of particles $\xi(\mathbb{R})=\infty$, when $\mathbb{K}_{\mathcal{A}}^{\xi \cap[-L, L]}$ converges to a continuous function as $L \rightarrow \infty$, the limit is written as $\mathbb{K}_{\mathcal{A}}^{\xi}$. If $\mathbb{P}_{\mathcal{A}}^{\xi \cap[-L, L]}$ converges to a probability measure $\mathbb{P}_{\mathcal{A}}^{\xi}$ on $\mathfrak{M}^{[0, \infty)}$, which is determinantal with the correlation kernel $\mathbb{K}_{\mathcal{A}}^{\xi}$, weakly in the sense of finite dimensional distributions as $L \rightarrow \infty$ in the vague topology, we say that the process $\left(\Xi_{\mathcal{A}}(t), \mathbb{P}_{\mathcal{A}}^{\xi}\right)$ is well defined with the correlation kernel $\mathbb{K}_{\mathcal{A}}^{\xi}$. (The regularity of the sample paths of $\Xi_{\mathcal{A}}(t)$ will be discussed in the forthcoming paper [14].) We will give sufficient conditions for initial configurations $\xi \in \mathfrak{M}_{0}$ so that the process $\left(\Xi_{\mathcal{A}}(t), \mathbb{P}_{\mathcal{A}}^{\xi}\right)$ is well defined (Theorem $[2.5)$. We denote by $\mathfrak{X}^{\mathcal{A}}$ the set of configurations $\xi$ satisfying the conditions and put $\mathfrak{X}_{0}^{\mathcal{A}}=\mathfrak{X}^{\mathcal{A}} \cap \mathfrak{M}_{0}$. It is clear that the configuration $\xi_{\mathcal{A}} \in \mathfrak{X}_{0}^{\mathcal{A}}$. Then, if we consider the finite particle systems $\Xi_{\mathcal{A}}(t)=\sum_{j=1}^{N} \delta_{Y_{j}(t)}, N \geq 2$, with (1.12) starting from the $N$-particle approximation of $\xi_{\mathcal{A}}$, (1.15), we can prove $\left(\Xi_{\mathcal{A}}(t), \mathbb{P}_{\mathcal{A}}^{\xi_{\mathcal{A}}^{N}}\right) \rightarrow\left(\Xi_{\mathcal{A}}(t), \mathbb{P}_{\text {Ai }}\right)$ as $N \rightarrow \infty$ in the sense of finite dimensional distributions (Theorem $2.6(\mathrm{i}))$. That is, $\left(\Xi_{\mathcal{A}}(t), \mathbb{P}_{\mathrm{Ai}}\right)=\left(\Xi_{\mathcal{A}}(t), \mathbb{P}_{\mathcal{A}}^{\xi_{\mathcal{A}}}\right)$ with (1.9). Moreover, we will show (1.36) and prove the relaxation phenomenon $\left(\Xi_{\mathcal{A}}(t+\theta), \mathbb{P}_{\mathrm{Ai}}\right) \rightarrow\left(\Xi_{\mathcal{A}}(t), \mathbf{P}_{\mathrm{Ai}}\right)$ (Theorem 2.6 (ii) $)$. 
The paper is organized as follows. In Sect. 2 preliminaries and main results are given. Some remarks on extensions of the present results are also given there. In Sect. 3 the properties of the Airy function used in this paper are summarized. Section 4 is devoted to proofs of results.

\section{Preliminaries and Main Results}

For $\xi(\cdot)=\sum_{j \in \Lambda} \delta_{x_{j}}(\cdot) \in \mathfrak{M}$, we introduce the following operations;

(shift) for $u \in \mathbb{R}, \tau_{u} \xi(\cdot)=\sum_{j \in \Lambda} \delta_{x_{j}+u}(\cdot)$,

(square) $\xi^{\langle 2\rangle}(\cdot)=\sum_{j \in \Lambda} \delta_{x_{j}^{2}}(\cdot)$

We use the convention such that

$$
\prod_{x \in \xi} f(x)=\exp \left\{\int_{\mathbb{R}} \xi(d x) \log f(x)\right\}=\prod_{x \in \operatorname{supp} \xi} f(x)^{\xi(\{x\})}
$$

for $\xi \in \mathfrak{M}$ and a function $f$ on $\mathbb{R}$, where $\operatorname{supp} \xi=\{x \in \mathbb{R}: \xi(\{x\})>0\}$. For a multivariate symmetric function $g$ we write $g\left((x)_{x \in \xi}\right)$ for $g\left(\left(x_{j}\right)_{j \in \Lambda}\right)$.

\subsection{Determinantal processes}

As an $\mathfrak{M}$-valued process $\left(\Xi(t), \mathbb{P}^{\xi}\right)$, we consider the system such that, for any integer $M \geq 1, f_{m} \in \mathrm{C}_{0}(\mathbb{R}), \theta_{m} \in \mathbb{R}, 1 \leq m \leq M, 0<t_{1}<\cdots<t_{M}<\infty$, the expectation of $\exp \left\{\sum_{m=1}^{M} \theta_{m} \int_{\mathbb{R}} f_{m}(x) \Xi\left(t_{m}, d x\right)\right\}$ can be expanded by

$$
\chi_{m}(x)=e^{\theta_{m} f_{m}(x)}-1, \quad 1 \leq m \leq M
$$

as

$$
\begin{aligned}
\mathcal{G}^{\xi}[\chi] \equiv \mathbb{E}^{\xi}\left[\exp \left\{\sum_{m=1}^{M} \theta_{m} \int_{\mathbb{R}} f_{m}(x) \Xi\left(t_{m}, d x\right)\right\}\right] \\
=\sum_{N_{1} \geq 0} \cdots \sum_{N_{M} \geq 0} \prod_{m=1}^{M} \frac{1}{N_{m} !} \int_{\mathbb{R}^{N_{1}}} \prod_{j=1}^{N_{1}} d x_{j}^{(1)} \cdots \int_{\mathbb{R}^{N_{M}}} \prod_{j=1}^{N_{M}} d x_{j}^{(M)} \\
\times \prod_{m=1}^{M} \prod_{j=1}^{N_{m}} \chi_{m}\left(x_{j}^{(m)}\right) \rho\left(t_{1}, \boldsymbol{x}^{(1)} ; \ldots ; t_{M}, \boldsymbol{x}^{(M)}\right) .
\end{aligned}
$$

Here $\rho$ 's are locally integrable functions, which are symmetric in the sense that $\rho\left(\ldots ; t_{m}, \sigma\left(\boldsymbol{x}^{(m)}\right) ; \ldots\right)=\rho\left(\ldots ; t_{m}, \boldsymbol{x}^{(m)} ; \ldots\right)$ with $\sigma\left(\boldsymbol{x}^{(m)}\right) \equiv\left(x_{\sigma(1)}^{(m)}, \ldots, x_{\sigma\left(N_{m}\right)}^{(m)}\right)$ for 
any permutation $\sigma \in \mathcal{S}_{N_{m}}, 1 \leq \forall m \leq M$. In such a system $\rho\left(t_{1}, \boldsymbol{x}^{(1)} ; \ldots ; t_{M}, \boldsymbol{x}^{(M)}\right)$ is called the $\left(N_{1}, \ldots, N_{M}\right)$-multitime correlation function and $\mathcal{G}^{\xi}[\chi]$ the generating function of multitime correlation functions. There are no multiple points with probability one for $t>0$. Then we assume that there is a function $\mathbb{K}(s, x ; t, y)$, which is continuous with respect to $(x, y) \in \mathbb{R}^{2}$ for any fixed $(s, t) \in[0, \infty)^{2}$, such that

$$
\rho\left(t_{1}, \boldsymbol{x}^{(1)} ; \ldots ; t_{M}, \boldsymbol{x}^{(M)}\right)=\operatorname{det}_{\substack{1 \leq j \leq N_{m}, 1 \leq k \leq N_{n} \\ 1 \leq m, n \leq M}}\left[\mathbb{K}\left(t_{m}, x_{j}^{(m)} ; t_{n}, x_{k}^{(n)}\right)\right]
$$

for any integer $M \geq 1$, any sequence $\left(N_{m}\right)_{m=1}^{M}$ of positive integers, and any time sequence $0<t_{1}<\cdots<t_{M}<\infty$. Let $\mathbf{T}=\left\{t_{1}, \ldots, t_{M}\right\}$. We note that $\Xi^{\mathbf{T}}=$ $\sum_{t \in \mathbf{T}} \delta_{t} \otimes \Xi(t)$ is a determinantal (Fermion) point process on $\mathbf{T} \times \mathbb{R}$ with an operator $\mathcal{K}$ given by

$$
\mathcal{K} f(s, x)=\sum_{t \in \mathbf{T}} \int_{\mathbb{R}} d y \mathbb{K}(s, x ; t, y) f(t, y), \quad f(t, \cdot) \in \mathrm{C}_{0}(\mathbb{R}), t \in \mathbf{T} .
$$

When $\mathcal{K}$ is symmetric, Soshnikov [24] and Shirai and Takahashi [23] gave sufficient conditions for $\mathbb{K}$ to be a correlation kernel of a determinantal point process. Though such conditions are not known for asymmetric cases, a variety of processes, which are determinantal with asymmetric correlation kernels, have been studied. See, for example, [29, 12. If there exists a function $\mathbb{K}$, which has the above properties and determines the finite dimensional distributions of the process $\left(\Xi(t), \mathbb{P}^{\xi}\right)$, we say the process $\left(\Xi(t), \mathbb{P}^{\xi}\right)$ is determinantal with the correlation kernel $\mathbb{K}[13]$.

For $N \in \mathbb{N}$, the determinant of an $N \times N$ matrix $\mathrm{M}=\left(m_{j k}\right)_{1 \leq j, k \leq N}$ is defined by $\sum_{\sigma \in \mathcal{S}_{N}} \operatorname{sgn}(\sigma) \prod_{j=1}^{N} m_{j \sigma(j)}$, where $\operatorname{sgn}(\sigma)$ denotes the sign of permutation $\sigma$. Any permutation $\sigma$ consists of exclusive cycles. If we write each cyclic permutation as

$$
\mathrm{c}=\left(\begin{array}{cccc}
a & b & \cdots & \omega \\
b & c & \cdots & a
\end{array}\right)
$$

and the number of cyclic permutations in a given $\sigma$ as $\ell(\sigma)$, then the determinant of $\mathrm{M}$ is expressed as

$$
\operatorname{det} \mathrm{M}=\sum_{\sigma \in \mathcal{S}_{N}} \operatorname{sgn}(\sigma) \prod_{c_{j}: 1 \leq j \leq \ell(\sigma)}\left(m_{a b} m_{b c} \ldots m_{\omega a}\right) .
$$

It implies that, with given $a_{1}, a_{2}, \ldots, a_{N}$, even if each element $m_{j k}$ of the matrix M is replaced by $m_{j k} \times\left(a_{j} / a_{k}\right)$, the value of determinant is not changed. The above observation will lead to the following lemma. 
Lemma 2.1 Let $(\Xi(t), \mathbb{P})$ and $(\widetilde{\Xi}(t), \widetilde{\mathbb{P}})$ be the processes, which are determinantal with correlation kernels $\mathbb{K}$ and $\widetilde{\mathbb{K}}$, respectively. If there is a function $G(s, x)$, which is continuous with respect to $x \in \mathbb{R}$ for any fixed $s \in[0, \infty)$, such that

$$
\mathbb{K}(s, x ; t, y)=\frac{G(s, x)}{G(t, y)} \widetilde{\mathbb{K}}(s, x ; t, y), \quad s, t \in[0, \infty), \quad x, y \in \mathbb{R},
$$

then

$$
(\Xi(t), \mathbb{P})=(\widetilde{\Xi}(t), \widetilde{\mathbb{P}})
$$

in the sense of finite dimensional distributions.

In literatures, (2.2) is called the gauge transformation and (2.3) is said to be the gauge invariance of the determinantal processes.

\subsection{The Weierstrass canonical product and entire functions}

For $\xi^{N} \in \mathfrak{M}_{0}, \xi^{N}(\mathbb{R})=N<\infty$, with $p \in \mathbb{N}_{0} \equiv \mathbb{N} \cup\{0\}$ we consider the product

$$
\Pi_{p}\left(\xi^{N}, z\right)=\prod_{x \in \xi^{N} \cap\{0\}^{c}} G\left(\frac{z}{x}, p\right), \quad z \in \mathbb{C},
$$

where

$$
G(u, p)=\left\{\begin{array}{lrl}
1-u & \text { if } \quad p=0 \\
(1-u) \exp \left[u+\frac{u^{2}}{2}+\cdots+\frac{u^{p}}{p}\right] & \text { if } \quad p \in \mathbb{N} .
\end{array}\right.
$$

The functions $G(u, p)$ are called the Weierstrass primary factors. With $\alpha>0$ we put

$$
M_{\alpha}\left(\xi^{N}\right)=\left(\int_{\{0\}^{\mathrm{c}}} \frac{1}{|x|^{\alpha}} \xi^{N}(d x)\right)^{1 / \alpha} .
$$

For $\xi \in \mathfrak{M}_{0}$ with $\xi(\mathbb{R})=\infty$, we write $M_{\alpha}(\xi, L)$ for $M_{\alpha}(\xi \cap[-L, L]), L>0$, and put $M_{\alpha}(\xi)=\lim _{L \rightarrow \infty} M_{\alpha}(\xi, L)$, if the limit finitely exists. If $M_{p+1}(\xi)<\infty$ for some $p \in \mathbb{N}_{0}$, the limit

$$
\Pi_{p}(\xi, z)=\lim _{L \rightarrow \infty} \Pi_{p}(\xi \cap[-L, L], z)=\prod_{x \in \xi \cap\{0\}^{c}} G\left(\frac{z}{x}, p\right), \quad z \in \mathbb{C}
$$

finitely exists. This infinite product is called the Weierstrass canonical product of genus $p$ [16]. The Hadamard theorem [16] claims that any entire function $f$ of finite order $\rho_{f}<\infty$ can be represented by

$$
f(z)=z^{m} e^{P_{q}(z)} \Pi_{p}\left(\xi_{f}, z\right),
$$


where $p$ is a nonnegative integer less than or equal to $\rho_{f}, P_{q}(z)$ is a polynomial in $z$ of degree $q \leq \rho_{f}, m$ is the multiplicity of the root at the origin, and $\xi_{f}=$ $\sum_{x \in f^{-1}(0) \cap\{0\}^{\mathrm{c}}} \delta_{x}$. We give two examples ;

$$
\begin{aligned}
\sin (\pi z) & =\pi z \Pi_{0}\left(\xi_{\mathbb{Z}}, z\right), \\
\operatorname{Ai}(z) & =e^{d_{0}+d_{1} z} \Pi_{1}\left(\xi_{\mathcal{A}}, z\right)
\end{aligned}
$$

with (1.17), (1.9) and

$$
\begin{aligned}
& d_{0}=\log \operatorname{Ai}(0)=-\log \left(3^{2 / 3} \Gamma(2 / 3)\right), \\
& d_{1}=\frac{\operatorname{Ai}^{\prime}(0)}{\operatorname{Ai}(0)}=-\frac{3^{1 / 3} \Gamma(2 / 3)}{\Gamma(1 / 3)}=-\frac{3^{5 / 6}(\Gamma(2 / 3))^{2}}{2 \pi} .
\end{aligned}
$$

For $\xi^{N} \in \mathfrak{M}_{0}$ with $\xi^{N}(\mathbb{R})=N$ we put

$$
\Phi_{p}\left(\xi^{N}, a, z\right) \equiv \Pi_{p}\left(\tau_{-a} \xi^{N}, z-a\right)=\prod_{x \in \xi^{N} \cap\{a\}^{c}} G\left(\frac{z-a}{x-a}, p\right), \quad a, z \in \mathbb{C} .
$$

With (1.15) we set

$$
\begin{aligned}
\Phi_{\mathcal{A}}\left(\xi^{N}, z\right) & \equiv e^{d_{1} z} \exp \left[\int_{\mathbb{R}} \frac{z}{x} \xi_{\mathcal{A}}^{N}(d x)\right] \Pi_{0}\left(\xi^{N}, z\right) \\
& =e^{d_{1} z} \exp \left[\int_{\{0\}^{\mathrm{c}}} \frac{z}{x}\left(\xi_{\mathcal{A}}^{N}-\xi^{N}\right)(d x)\right] \Pi_{1}\left(\xi^{N}, z\right), \quad z \in \mathbb{C}, \\
\Phi_{\mathcal{A}}\left(\xi^{N}, a, z\right) & \equiv \Phi_{\mathcal{A}}\left(\tau_{-a} \xi^{N}, z-a\right) \\
& =e^{d_{1}(z-a)} \exp \left[\int_{\mathbb{R}} \frac{z-a}{x} \xi_{\mathcal{A}}^{N}(d x)\right] \Phi_{0}\left(\xi^{N}, a, z\right), \quad a, z \in \mathbb{C} .
\end{aligned}
$$

Lemma 2.2 Let $\xi^{N} \in \mathfrak{M}_{0}$ with $\xi^{N}(\mathbb{R})=N<\infty$ and $\xi^{N}(\{0\})=0$. Then for $a \in \operatorname{supp} \xi^{N}, z \neq a$,

$$
\Phi_{\mathcal{A}}\left(\xi^{N}, a, z\right)=\frac{1}{z-a} \frac{\Phi_{\mathcal{A}}\left(\xi^{N}, z\right)}{\Phi_{\mathcal{A}}^{\prime}\left(\xi^{N}, a\right)},
$$

where $\Phi_{\mathcal{A}}^{\prime}(\cdot, a)=\partial \Phi_{\mathcal{A}}(\cdot, z) /\left.\partial z\right|_{z=a}$.

Proof. Since $1-(z-a) /(x-a)=(x-z) /(x-a)=(1-z / x) /(1-a / x)$,

$$
\Phi_{\mathcal{A}}\left(\xi^{N}, a, z\right)=\frac{e^{d_{1} z} \exp \left[\int_{\mathbb{R}} \frac{z}{x} \xi_{\mathcal{A}}^{N}(d x)\right] \Pi_{0}\left(\xi^{N}-\delta_{a}, z\right)}{e^{d_{1} a} \exp \left[\int_{\mathbb{R}} \frac{a}{x} \xi_{\mathcal{A}}^{N}(d x)\right] \Pi_{0}\left(\xi^{N}-\delta_{a}, a\right)},
$$


where the numerator is equal to $\Phi_{\mathcal{A}}\left(\xi^{N}, z\right) /(1-z / a)$. From (2.11), we have

$$
\begin{aligned}
& \frac{\partial}{\partial z} \Phi_{\mathcal{A}}\left(\xi^{N}, z\right)=d_{1} \Phi_{\mathcal{A}}\left(\xi^{N}, z\right) \\
& \quad+e^{d_{1} z} \int_{\mathbb{R}} \frac{1}{x} \xi_{\mathcal{A}}^{N}(d x) \exp \left[\int_{\mathbb{R}} \frac{z}{y} \xi_{\mathcal{A}}^{N}(d y)\right] \Pi_{0}\left(\xi^{N}, z\right) \\
& \quad+e^{d_{1} z} \exp \left[\int_{\mathbb{R}} \frac{z}{y} \xi_{\mathcal{A}}^{N}(d y)\right] \int_{\mathbb{R}}\left(-\frac{1}{x}\right) \Pi_{0}\left(\xi^{N}-\delta_{x}, z\right) \xi^{N}(d x) .
\end{aligned}
$$

Since $a \in \operatorname{supp} \xi^{N}$ is assumed,

$$
\Phi_{\mathcal{A}}^{\prime}\left(\xi^{N}, a\right)=e^{d_{1} a} \exp \left[\int_{\mathbb{R}} \frac{a}{x} \xi^{N}(d x)\right]\left(-\frac{1}{a}\right) \Pi_{0}\left(\xi^{N}-\delta_{a}, a\right) .
$$

It implies that the denominator of (2.14) is equal to $-a \Phi_{\mathcal{A}}^{\prime}\left(\xi^{N}, a\right)$. Then (2.13) is obtained.

For $\xi^{N} \in \mathfrak{M}_{0}$ with $\xi^{N}(\mathbb{R})=N$ we put

$$
M_{\mathcal{A}}\left(\xi^{N}\right)=\int_{\{0\}^{\mathrm{c}}} \frac{1}{x}\left(\xi_{\mathcal{A}}^{N}-\xi^{N}\right)(d x) .
$$

For $\xi \in \mathfrak{M}_{0}$ with $\xi(\mathbb{R})=\infty$ we write $M_{\mathcal{A}}(\xi, L)$ for $M_{\mathcal{A}}(\xi \cap[-L, L]), L>0$, and put $M_{\mathcal{A}}(\xi)=\lim _{L \rightarrow \infty} M_{\mathcal{A}}(\xi, L)$, if the limit finitely exists. For $\xi \in \mathfrak{M}_{0}, p \in$ $\mathbb{N}_{0}, a \in \mathbb{R}$, and $z \in \underset{L \rightarrow \infty}{\mathbb{C}}$ we define $\Phi_{p}(\xi, a, z)=\lim _{L \rightarrow \infty} \Phi_{p}(\xi \cap[a-L, a+L], a, z)$ and $\Phi_{\mathcal{A}}(\xi, a, z)=\lim _{L \rightarrow \infty} \Phi_{\mathcal{A}}(\xi \cap[a-L, a+L], a, z)$, if the limits finitely exist. We note that $\Phi_{p}(\xi, a, z)$ finitely exists and is not identically 0 , if $M_{p+1}\left(\tau_{-a} \xi\right)<\infty$, and $\Phi_{\mathcal{A}}(\xi, a, z)$ does and $\Phi_{\mathcal{A}}(\xi, a, z) \not \equiv 0$, if $\left|M_{\mathcal{A}}\left(\tau_{-a} \xi\right)\right|<\infty$ and $M_{2}\left(\tau_{-a} \xi\right)<\infty$. For $\xi \in \mathfrak{M}_{0}, a \in \operatorname{supp} \xi$, the following equalities will hold, if all the entries of them finitely exist ;

$$
\begin{aligned}
& \Phi_{0}(\xi, a, z)=\Pi_{0}(\xi, z) \Phi_{0}\left(\xi \cap\{0\}^{\mathrm{c}}, a, 0\right)\left(\frac{z}{a}\right)^{\xi(\{0\})} \frac{a}{a-z}, \\
& \Phi_{0}\left(\xi \cap\{0\}^{\mathrm{c}}, a, 0\right)=\Pi_{0}\left(\xi \cap\{-a\}^{\mathrm{c}},-a\right) \Phi_{0}\left(\xi^{\langle 2\rangle} \cap\{0\}^{\mathrm{c}}, a^{2}, 0\right) 2^{1-\xi(\{-a\})}
\end{aligned}
$$

and then

$$
\begin{aligned}
\Phi_{1}(\xi, a, z)= & e^{S(\xi, a, z)} \Pi_{1}(\xi, z) \Pi_{1}\left(\xi \cap\{-a\}^{\mathrm{c}},-a\right) \\
& \times \Phi_{0}\left(\xi^{\langle 2\rangle} \cap\{0\}^{\mathrm{c}}, a^{2}, 0\right)\left(\frac{z}{a}\right)^{\xi(\{0\})} \frac{a}{a-z}
\end{aligned}
$$

where

$$
S(\xi, a, z)=\int_{\{a\}^{\mathrm{c}}} \frac{z-a}{x-a} \xi(d x)-\int_{\{0\}^{\mathrm{c}}} \frac{z}{x} \xi(d x)+\int_{\{0,-a\}^{\mathrm{c}}} \frac{a}{x} \xi(d x) .
$$


Lemma 2.3 For $a \in \mathcal{A}, z \neq a$

$$
\frac{1}{z-a} \frac{\operatorname{Ai}(z)}{\operatorname{Ai}^{\prime}(a)}=\Phi_{\mathcal{A}}\left(\xi_{\mathcal{A}}, a, z\right) .
$$

Proof. By (2.8) and the definition (2.11),

$$
\operatorname{Ai}(z)=e^{d_{0}} \Phi_{\mathcal{A}}\left(\xi_{\mathcal{A}}, z\right), \quad z \in \mathbb{C} .
$$

As approximations of the Airy function we introduce functions

$$
\operatorname{Ai}_{N}(z)=e^{d_{0}+d_{1} z} \prod_{\ell=1}^{N}\left(1-\frac{z}{a_{\ell}}\right) e^{z / a_{\ell}}, \quad N \in \mathbb{N}
$$

where $0>a_{1}>\cdots>a_{N}$ are the first $N$ zeros of $\operatorname{Ai}(z)$. Since $\xi_{\mathcal{A}}^{N}=\sum_{j=1}^{N} \delta_{a_{j}}$ satisfies the condition of Lemma 2.2, (2.13) with (2.19) and $\operatorname{Ai}_{N}^{\prime}\left(a_{j}\right)=e^{d_{0}} \Phi_{\mathcal{A}}^{\prime}\left(\xi_{\mathcal{A}}^{N}, a_{j}\right)$ gives

$$
\frac{1}{z-a_{j}} \frac{\mathrm{Ai}_{N}(z)}{\operatorname{Ai}_{N}^{\prime}\left(a_{j}\right)}=\Phi_{\mathcal{A}}\left(\xi_{\mathcal{A}}^{N}, a_{j}, z\right), \quad 1 \leq j \leq N .
$$

Taking $N \rightarrow \infty$, we have (2.18).

\subsection{Statement of results}

For the solution $\boldsymbol{X}(t)=\left(X_{1}(t), X_{2}(t), \ldots, X_{N}(t)\right)$ of Dyson's model (1.1) with $\beta=2$ with the initial state $\boldsymbol{X}(0)=\boldsymbol{x}$, we denote the distribution of the process $\Xi(t)=$ $\sum_{j=1}^{N} \delta_{X_{j}(t)}$ by $\mathbb{P}^{\xi^{N}}$ with $\xi^{N}=\sum_{j=1}^{N} \delta_{x_{j}}$. In [13] we proved that Dyson's model (1.1) with $\beta=2$ starting from any fixed configuration $\xi^{N} \in \mathfrak{M}$ is determinantal with the correlation kernel $\mathbb{K}^{\xi^{N}}$ given by

$$
\begin{aligned}
& \mathbb{K}^{\xi^{N}}(s, x ; t, y)= \frac{1}{2 \pi \sqrt{-1}} \oint_{\Gamma\left(\xi^{N}\right)} d z \int_{\sqrt{-1} \mathbb{R}} \frac{d w}{\sqrt{-1}} \\
& \times p_{\sin }(s, x-z) \frac{1}{w-z} \prod_{x^{\prime} \in \xi^{N}}\left(1-\frac{w-z}{x^{\prime}-z}\right) p_{\sin }(-t, w-y) \\
&-\mathbf{1}(s>t) p_{\sin }(s-t, y-x)
\end{aligned}
$$

where $\Gamma\left(\xi^{N}\right)$ is a closed contour on the complex plane $\mathbb{C}$ encircling the points in $\operatorname{supp} \xi^{N}$ on $\mathbb{R}$ once in the positive direction, and $p_{\text {sin }}$ is given by (1.20). If $\xi^{N} \in \mathfrak{M}_{0}$, by performing the Cauchy integrals (2.21) is written as

$$
\begin{aligned}
\mathbb{K}^{\xi^{N}}(s, x ; t, y)= & \int_{\mathbb{R}} \xi^{N}\left(d x^{\prime}\right) \int_{\sqrt{-1} \mathbb{R}} \frac{d y^{\prime}}{\sqrt{-1}} p_{\sin }\left(s, x-x^{\prime}\right) \Phi_{0}\left(\xi^{N}, x^{\prime}, y^{\prime}\right) p_{\sin }\left(-t, y^{\prime}-y\right) \\
& -\mathbf{1}(s>t) p_{\sin }(s-t, x-y) .
\end{aligned}
$$

Then the following is obtained for the process $\left(\Xi_{\mathcal{A}}(t), \mathbb{P}_{\mathcal{A}}^{\xi^{N}}\right)$ with $\Xi_{\mathcal{A}}(t)=\sum_{j=1}^{N} \delta_{Y_{j}(t)}$, where $\boldsymbol{Y}(t)=\left(Y_{1}(t), \ldots, Y_{N}(t)\right)$ is given by (1.12). 
Proposition 2.4 The process $\left(\Xi_{\mathcal{A}}(t), \mathbb{P}_{\mathcal{A}}^{\xi^{N}}\right)$, starting from any fixed configuration $\xi^{N} \in \mathfrak{M}_{0}$ with $\xi^{N}(\mathbb{R})=N<\infty$, is determinantal with the correlation kernel $\mathbb{K}_{\mathcal{A}}^{\xi^{N}}$ given by

$$
\begin{aligned}
\mathbb{K}_{\mathcal{A}}^{\xi^{N}}(s, x ; t, y)= & \int_{\mathbb{R}} \xi^{N}\left(d x^{\prime}\right) \int_{\sqrt{-1} \mathbb{R}} \frac{d y^{\prime}}{\sqrt{-1}} q\left(0, s, x-x^{\prime}\right) \Phi_{\mathcal{A}}\left(\xi^{N}, x^{\prime}, y^{\prime}\right) q\left(t, 0, y^{\prime}-y\right) \\
& -\mathbf{1}(s>t) q(t, s, x-y),
\end{aligned}
$$

where $q$ is given by (1.30).

We introduce the following conditions:

(C.1) there exists $C_{0}>0$ such that $\left|M_{\mathcal{A}}(\xi)\right|<C_{0}$,

(C.2) (i) there exist $\alpha \in(3 / 2,2)$ and $C_{1}>0$ such that $M_{\alpha}(\xi) \leq C_{1}$,

(ii) there exist $\beta>0$ and $C_{2}>0$ such that

$$
M_{1}\left(\tau_{-a^{2}} \xi^{\langle 2\rangle}\right) \leq C_{2}(|a| \vee 1)^{-\beta} \text { for all } a \in \operatorname{supp} \xi .
$$

We denote by $\mathfrak{X}^{\mathcal{A}}$ the set of configurations $\xi$ satisfying the conditions $(\mathbf{C . 1})$ and (C.2), and put $\mathfrak{X}_{0}^{\mathcal{A}}=\mathfrak{X}^{\mathcal{A}} \cap \mathfrak{M}_{0}$.

Theorem 2.5 If $\xi \in \mathfrak{X}_{0}^{\mathcal{A}}$, the process $\left(\Xi_{\mathcal{A}}(t), \mathbb{P}_{\mathcal{A}}^{\xi}\right)$ is well defined with the correlation kernel

$$
\begin{aligned}
\mathbb{K}_{\mathcal{A}}^{\xi}(s, x ; t, y)= & \int_{\mathbb{R}} \xi\left(d x^{\prime}\right) \int_{\sqrt{-1} \mathbb{R}} \frac{d y^{\prime}}{\sqrt{-1}} q\left(0, s, x-x^{\prime}\right) \Phi_{\mathcal{A}}\left(\xi, x^{\prime}, y^{\prime}\right) q\left(t, 0, y^{\prime}-y\right) \\
& -\mathbf{1}(s>t) q(t, s, x-y) .
\end{aligned}
$$

In the proof of this theorem, a useful estimate of $\Phi_{\mathcal{A}}$ in (2.24) is obtained (Lemma 4.3 (ii)). By virtue of it, we can see

$$
\mathbb{K}_{\mathcal{A}}^{\xi}(t, x ; t, y) \mathbb{K}_{\mathcal{A}}^{\xi}(t, y ; t, x) d x d y \rightarrow \xi(d x) \mathbf{1}(x=y) \quad \text { as } \quad t \rightarrow 0
$$

in the vague topology. Then Theorem 2.5 gives an infinite particle system starting form the configuration $\xi$.

The main result of the present paper is the following.

Theorem 2.6 (i) $\operatorname{Let} \xi_{\mathcal{A}}^{N}(\cdot)$ be the configuration (1.15). Then

$$
\left(\Xi_{\mathcal{A}}(t), \mathbb{P}_{\mathcal{A}}^{\xi_{\mathcal{A}}^{N}}\right) \rightarrow\left(\Xi_{\mathcal{A}}(t), \mathbb{P}_{\mathrm{Ai}}\right) \quad \text { as } \quad N \rightarrow \infty
$$

in the sense of finite dimensional distributions. Here the process $\left(\Xi_{\mathcal{A}}(t), \mathbb{P}_{\mathrm{Ai}}\right)$ is determinantal with the correlation kernel (1.23) with setting (1.26) and $p_{f}(s, x ; t, y)=$ $p_{\text {Ai }}(t-s, y \mid x)$ with (1.31), that is

$$
\begin{aligned}
\mathbb{K}_{\mathrm{Ai}}(s, x ; t, y)= & \sum_{a \in \mathrm{Ai}^{-1}(0)} \int_{\sqrt{-1}} \frac{d z}{\mathbb{R}} p_{\mathrm{Ai}}(s, x \mid a) \frac{1}{z-a} \frac{\operatorname{Ai}(z)}{\mathrm{Ai}^{\prime}(a)} p_{\mathrm{Ai}}(-t, z \mid y) \\
& -\mathbf{1}(s>t) p_{\mathrm{Ai}}(s-t, x \mid y)
\end{aligned}
$$


(ii) Let $\left(\Xi_{\mathcal{A}}(t), \mathbf{P}_{\mathrm{Ai}}\right)$ be the process, which is determinantal with the extended Airy kernel (1.37). Then

$$
\left(\Xi_{\mathcal{A}}(t+\theta), \mathbb{P}_{\mathrm{Ai}}\right) \rightarrow\left(\Xi_{\mathcal{A}}(t), \mathbf{P}_{\mathrm{Ai}}\right) \quad \text { as } \quad \theta \rightarrow \infty
$$

weakly in the sense of finite dimensional distributions.

\subsection{Remarks on Extensions of the Results}

(1) By definition (2.15),$M_{\mathcal{A}}\left(\xi_{\mathcal{A}}\right)=0$. The asymptotic property of the zeros (1.11) implies

$$
\zeta^{\mathcal{A}}(\alpha) \equiv\left(M_{\alpha}\left(\xi_{\mathcal{A}}\right)\right)^{\alpha}=\sum_{a \in \mathcal{A}} \frac{1}{|a|^{\alpha}}<\infty, \quad \text { if } \quad \alpha>\widehat{\rho}_{f}=\frac{3}{2}
$$

In general, order of growth $\rho_{f}$ of a canonical produce (2.5) is equal to the convergence exponent $\widehat{\rho}_{f}$ of the sequence of its zeros [16]. For $\operatorname{Ai}(z), \rho_{f}=3 / 2$. The function $\zeta^{\mathcal{A}}(\alpha)$ may be called the Airy zeta function [30], which is meromorphic in the whole of $\mathbb{C}$ [7]. Moreover, we know

$$
\zeta^{\mathcal{A}}(2)=M_{1}\left(\xi_{\mathcal{A}}^{\langle 2\rangle}\right)=\sum_{a \in \mathcal{A}} \frac{1}{a^{2}}=d_{1}^{2}<\infty
$$

with (2.9). Then $\xi_{\mathcal{A}}$ satisfies the conditions (C.1) and (C.2) : $\xi_{\mathcal{A}} \in \mathfrak{X}^{\mathcal{A}}$. Since $\xi_{\mathcal{A}} \in \mathfrak{M}_{0}$, Theorem 2.5 guarantees the well-definedness of the infinite particle system $\left(\Xi_{\mathcal{A}}(t), \mathbb{P}_{\mathcal{A}}^{\xi_{\mathcal{A}}}\right)$. (Its equivalence with $\left(\Xi(t), \mathbb{P}_{\mathrm{Ai}}\right)$ is stated in Theorem 2.6 (i).) Note that the negative divergence (1.16) of the drift term $D_{\mathcal{A}_{N}} t$ of (1.12) in $N \rightarrow \infty$ for $t<\infty$ corresponds to that $\zeta^{\mathcal{A}}(1)=-\sum_{a \in \mathcal{A}}(1 / a)=\infty$. This fact and (2.29) mean that the Airy function has genus 1 [16, 30].

Examples of infinite particle configurations in $\mathfrak{X}_{0}^{\mathcal{A}}$ other than $\xi_{\mathcal{A}}$ are given as follows. For $\kappa>0$, we put

$$
g^{\kappa}(x)=\operatorname{sgn}(x)|x|^{\kappa}, x \in \mathbb{R}, \text { and } \eta^{\kappa}(\cdot)=\sum_{\ell \in \mathbb{Z}} \delta_{g^{\kappa}(\ell)}(\cdot) .
$$

For any $\kappa>1 / 2$ we can confirm by simple calculation that any configuration $\xi \in \mathfrak{M}_{0}$ with supp $\xi \subset \operatorname{supp} \eta^{\kappa}=\left\{g^{\kappa}(\ell): \ell \in \mathbb{Z}\right\}$ satisfies (C.2)(i) with any $\alpha \in(1 / \kappa, 2)$ and some $C_{1}=C_{1}(\alpha)>0$ depending on $\alpha$ and (C.2)(ii) with any $\beta \in(0,2 \kappa-1)$ and some $C_{2}=C_{2}(\beta)>0$ depending on $\beta$. Assume that $\xi \in \mathfrak{M}_{0}$ is chosen so that $\operatorname{supp} \xi \subset \operatorname{supp} \eta^{\kappa}$ for some $\kappa>1 / 2$ and $\left|M_{\mathcal{A}}(\xi)\right|<\infty$. Then $\xi \in \mathfrak{X}_{0}^{\mathcal{A}}$. The fact (1.11) implies that this assumption can be satisfied only if $\kappa \in(1 / 2,2 / 3]$.

(2) If there exists, however, $\beta^{\prime}<(\beta-1) \wedge(\beta / 2)$ for $\xi \in \mathfrak{M}_{0}$ such that $\sharp\{x \in \xi$ : $\left.\xi\left(\left[x-|x|^{\beta^{\prime}}, x+|x|^{\beta^{\prime}}\right]\right) \geq 2\right\}=\infty$, then $\xi$ does not satisfy the condition (C.2) (ii). In order to include such initial configurations as well as those with multiple points in 
our study of the process $\left(\Xi_{\mathcal{A}}(t), \mathbb{P}_{\mathcal{A}}^{\xi}\right)$ with $\xi(\mathbb{R})=\infty$, we introduce another condition for configurations:

(C.3) there exists $\kappa \in(1 / 2,2 / 3]$ and $m \in \mathbb{N}$ such that

$$
m(\xi, \kappa) \equiv \max _{k \in \mathbb{Z}} \xi\left(\left[g^{\kappa}(k), g^{\kappa}(k+1)\right]\right) \leq m .
$$

We denote by $\mathfrak{Y}_{\kappa, m}^{\mathcal{A}}$ the set of configurations $\xi$ satisfying $(\mathbf{C . 1})$ and (C.3) with $\kappa \in(1 / 2,2 / 3]$ and $m \in \mathbb{N}$, and put

$$
\mathfrak{Y}^{\mathcal{A}}=\bigcup_{\kappa \in(1 / 2,2 / 3]} \bigcup_{m \in \mathbb{N}} \mathfrak{Y}_{\kappa, m}^{\mathcal{A}} .
$$

Noting that the set $\{\xi \in \mathfrak{M}: m(\xi, \kappa) \leq m\}$ is relatively compact for each $\kappa \in$ $(1 / 2,2 / 3]$ and $m \in \mathbb{N}$, we see that $\mathfrak{Y}^{\mathcal{A}}$ is locally compact.

In the present paper, we report our study of the relaxation process $\left(\Xi(t), \mathbb{P}_{\mathrm{Ai}}\right)$ from a special initial configuration $\xi_{\mathcal{A}}$ to the stationary state $\mu_{\mathrm{Ai}}$. We expect that $\mu_{\mathrm{Ai}}$ is an attractor in the configuration space $\mathfrak{Y}^{\mathcal{A}}$ and $\xi_{\mathcal{A}}$ is a point included in the basin. Motivated by such consideration, we are interested in the continuity of the process with respect to initial configuration. We have found, however, that if $\xi(\mathbb{R})=\infty$, the weak convergence of processes in the sense of finite dimensional distributions can not be concluded from the convergence of initial configurations in the vague topology. Following the idea given by our previous paper [13], we introduce a stronger topology for $\mathfrak{Y}^{\mathcal{A}}$. if

Suppose that $\xi, \xi_{n} \in \mathfrak{Y}^{\mathcal{A}}, n \in \mathbb{N}$. We say that $\xi_{n}$ converges $\Phi_{\mathcal{A}}$-moderately to $\xi$,

$$
\lim _{n \rightarrow \infty} \Phi_{\mathcal{A}}\left(\xi_{n}, \sqrt{-1}, \cdot\right)=\Phi_{\mathcal{A}}(\xi, \sqrt{-1}, \cdot) \text { uniformly on any compact set of } \mathbb{C} .
$$

It is easy to see that (2.30) is satisfied, if the following two conditions hold:

$$
\begin{aligned}
& \lim _{L \rightarrow \infty} \sup _{n>0} \lim _{M \rightarrow \infty}\left|M_{\mathcal{A}}\left(\xi_{n}, M\right)-M_{\mathcal{A}}\left(\xi_{n}, L\right)\right|=0, \\
& \lim _{L \rightarrow \infty} \sup _{n>0}\left|M_{2}\left(\xi_{n}\right)-M_{2}\left(\xi_{n}, L\right)\right|=0 .
\end{aligned}
$$

By the similar argument given in [13], the following statements are proved.

(i) If $\xi \in \mathfrak{Y}^{\mathcal{A}}$, the process $\left(\Xi_{\mathcal{A}}, \mathbb{P}_{\mathcal{A}}^{\xi}\right)$ is well defined.

(ii) Suppose that $\xi, \xi_{n} \in \mathfrak{Y}_{\kappa, n}^{\mathcal{A}}, n \in \mathbb{N}$, for some $\kappa \in(1 / 2,2 / 3]$ and $m \in \mathbb{N}$. If $\xi_{n}$ converges $\Phi_{\mathcal{A}}$-moderately to $\xi$, then $\left(\Xi_{\mathcal{A}}, \mathbb{P}_{\mathcal{A}}^{\xi_{n}}\right) \rightarrow\left(\Xi_{\mathcal{A}}, \mathbb{P}_{\mathcal{A}}^{\xi}\right)$ weakly in the sense of finite dimensional distributions as $n \rightarrow \infty$ in the vague topology.

Moreover, we can show $\mu_{\mathrm{Ai}}\left(\mathfrak{Y}^{\mathcal{A}}\right)=1$. By this fact and the above mentioned continuity with respect to initial configurations, we can prove that the stationary 
process $\left(\Xi_{\mathcal{A}}(t), \mathbf{P}_{\mathrm{Ai}}\right)$, which is determinantal with the extended Airy kernel (1.37), is Markovian [14].

(3) As mentioned in Introduction, the purpose of the present paper is to give a method for asymmetric initial configurations to construct infinite particle systems of Brownian motions interacting through pair force $1 / x$. In order to clarify the results, we have concentrated on the case in this paper such that the initial configuration is $\xi_{\mathcal{A}}$ (Theorem 2.6) or its modification $\xi \in \mathfrak{X}_{0}^{\mathcal{A}}$; see the condition (C.1) with (2.15) (Theorem 2.5). In the former case the constructed infinite particle system $\left(\Xi_{\mathcal{A}}(t), \mathbb{P}_{\mathrm{Ai}}\right)$ has the stationary measure $\mu_{\mathrm{Ai}}$, which is obtained in the soft-edge scaling limit of the eigenvalue distribution in GUE well-studied in the random matrix theory. Thus we have specified the entire function used in our analysis in the form $\Phi_{\mathcal{A}}(\xi, z)$ given by (2.11), which is suitable for the Airy function (see (2.19)). The point of our method is to put the relationship between the entire function appearing in the correlation kernel $\mathbb{K}^{\xi}$, the "typical" initial configuration $\xi_{f}$, and the drift term in the SDEs providing finite-particle approximations. By the same argument as reported here, the following will be proved. Let $f$ be the entire function such that $f(0) \neq 0$, it is expressed by the Weierstrass canonical product of genus one, $\Pi_{1}\left(\xi_{f}, z\right)$, and the zeros can be labelled as $0<\left|x_{1}\right|<\left|x_{2}\right|<\cdots$. Then with $\xi_{f}=\sum_{j=1}^{\infty} \delta_{x_{j}}$ we put

$$
D\left(\xi_{f}, N\right)=\sum_{j=1}^{N} \frac{1}{x_{j}}
$$

and introduce the $N$-particle system

$$
Y_{j}^{f}(t)=X_{j}(t)+D\left(\xi_{f}, N\right) t, \quad 1 \leq j \leq N, \quad t \in[0, \infty)
$$

where $\boldsymbol{X}(t)=\left(X_{1}(t), \ldots, X_{N}(t)\right)$ is the solution of Dyson's model (1.1) starting from the first $N$ zeros of $f, X_{j}(0)=x_{j}, 1 \leq j \leq N$. Then $\Xi_{f}(t)=\sum_{j=1}^{N} \delta_{Y_{j}^{f}(t)}$ converges to the dynamics in $N \rightarrow \infty$, in the sense of finite dimensional distribution, which is determinantal with the correlation kernel

$$
\begin{aligned}
\mathbb{K}_{f}^{\xi_{f}}(s, x ; t, y)= & \int_{\mathbb{R}} \xi_{f}\left(d x^{\prime}\right) \int_{\sqrt{-1} \mathbb{R}} \frac{d y^{\prime}}{\sqrt{-1}} p_{\sin }\left(s, x-x^{\prime}\right) \Phi_{1}\left(\xi_{f}, x^{\prime}, y^{\prime}\right) p_{\sin }\left(-t, y^{\prime}-y\right) \\
& -\mathbf{1}(s>t) p_{\sin }(s-t, x-y) .
\end{aligned}
$$

Moreover, even if the initial configuration $\xi$ is different from $\xi_{f}$, but it satisfies the condition

$$
\left|\int_{-L}^{L} \frac{1}{x}\left(\xi_{f}-\xi\right)(d x)\right|<C_{0}
$$

for any $L>0$ with a positive finite $C_{0}$ independent of $L$, then the process starting from $\xi$, is well-defined. In general, the obtained dynamics with an infinite number of particles is not stationary, while Theorem 2.6 gave the example which converges to a stationary dynamics $\left(\Xi_{\mathcal{A}}(t), \mathbf{P}_{\mathrm{Ai}}\right)$ in the long-term limit. 


\section{Properties of the Airy Functions}

\subsection{Integrals}

By the fact $\operatorname{Ai}^{\prime \prime}(x)=x \operatorname{Ai}(x)$, the following primitive is obtained for $c \neq 0[30$,

$$
\begin{gathered}
\int d u(\operatorname{Ai}(c(u+x)))^{2}=(u+x)(\operatorname{Ai}(c(u+x)))^{2}-\frac{1}{c}\left(\operatorname{Ai}^{\prime}(c(u+x))\right)^{2}, \\
\int d u \operatorname{Ai}(c(u+x)) \operatorname{Ai}(c(u+y)) \\
=\frac{\operatorname{Ai}^{\prime}(c(u+x)) \operatorname{Ai}(c(u+y))-\operatorname{Ai}(c(u+x)) \operatorname{Ai}^{\prime}(c(u+y))}{c^{2}(x-y)} .
\end{gathered}
$$

By setting $c=1$ and integral interval be $[0, \infty)$ in (3.2), we obtain the integral

$$
\int_{0}^{\infty} d u \operatorname{Ai}(u+x) \operatorname{Ai}(u+y)=\frac{\operatorname{Ai}(x) \operatorname{Ai}^{\prime}(y)-\operatorname{Ai}^{\prime}(x) \operatorname{Ai}(y)}{x-y},
$$

since $\lim _{x \rightarrow \infty} \operatorname{Ai}(x)=\lim _{x \rightarrow \infty} \operatorname{Ai}^{\prime}(x)=0$ by (1.5). If we set $y=a \in \mathcal{A}$ and $x=z \neq a$, then

$$
\int_{0}^{\infty} d u \operatorname{Ai}(u+z) \operatorname{Ai}(u+a)=\frac{\operatorname{Ai}(z) \operatorname{Ai}^{\prime}(a)}{z-a},
$$

since $\operatorname{Ai}(a)=0$. Then we have the expression

$$
\frac{1}{z-a} \frac{\operatorname{Ai}(z)}{\operatorname{Ai}^{\prime}(a)}=\frac{1}{\left(\operatorname{Ai}^{\prime}(a)\right)^{2}} \int_{0}^{\infty} d u \operatorname{Ai}(u+z) \operatorname{Ai}(u+a)
$$

for $a \in \mathcal{A}, z \neq a$.

\subsection{Airy transform}

The following integral formulas are proved [11].

Lemma 3.1 For $c>0, x, y \in \mathbb{R}$

$$
\begin{aligned}
& \int_{\mathbb{R}} d u e^{c u} \operatorname{Ai}(u+x) \operatorname{Ai}(u+y)=\frac{1}{\sqrt{4 \pi c}} e^{-(x-y)^{2} /(4 c)-c(x+y) / 2+c^{3} / 12}, \\
& \int_{\mathbb{R}} d y \int_{\mathbb{R}} d u e^{c u} \operatorname{Ai}(u+x) \operatorname{Ai}(u+y)=e^{-c x+c^{3} / 3} .
\end{aligned}
$$

Proof. Consider the integral

$$
\begin{aligned}
I & =\int_{\mathbb{R}} d u e^{c x} \operatorname{Ai}(u+x) \operatorname{Ai}(u+y) \\
& =\int_{0}^{\infty} d u e^{c u} \operatorname{Ai}(u+x) \operatorname{Ai}(u+y)+\int_{-\infty}^{0} d u e^{c u} \operatorname{Ai}(u+x) \operatorname{Ai}(u+y) .
\end{aligned}
$$


By the definition of the Airy function (1.4), for any $\eta>0$,

$$
\begin{aligned}
I & =\int_{0}^{\infty} d u e^{c u} \frac{1}{(2 \pi)^{2}} \int_{\Im z=\eta} d z \int_{\Im w>c-\eta} d w e^{\sqrt{-1}\left\{z^{3} / 3+(u+x) z+w^{3} / 3+(y+u) w\right\}} \\
& +\int_{-\infty}^{0} d u e^{c u} \frac{1}{(2 \pi)^{2}} \int_{\Im z=\eta} d z \int_{\Im w<c-\eta} d w e^{\sqrt{-1}\left\{z^{3} / 3+(u+x) z+w^{3} / 3+(u+y) w\right\}} \\
& =\frac{1}{(2 \pi)^{2}} \int_{\Im z=\eta} d z \int_{\Im w>c-\eta} d w e^{\sqrt{-1}\left(z^{3} / 3+x z+w^{3} / 3+y w\right)} \int_{0}^{\infty} d u e^{\{c+\sqrt{-1}(z+w)\} u} \\
& +\frac{1}{(2 \pi)^{2}} \int_{\Im z=\eta} d z \int_{\Im w<c-\eta} d w e^{\sqrt{-1}\left(z^{3} / 3+x z+w^{3} / 3+y w\right)} \int_{-\infty}^{0} d u e^{\{c+\sqrt{-1}(z+w)\} u} \\
& =-\frac{1}{(2 \pi)^{2}} \int_{\Im z=\eta} d z \int_{\Im w>c-\eta} d w e^{\sqrt{-1}\left(z^{3} / 3+x z+w^{3} / 3+y w\right)} \frac{1}{c+\sqrt{-1}(z+w)} \\
& +\frac{1}{(2 \pi)^{2}} \int_{\Im z=\eta} d z \int_{\Im w<c-\eta} d w e^{\sqrt{-1}\left(z^{3} / 3+x z+w^{3} / 3+y w\right)} \frac{1}{c+\sqrt{-1}(z+w)} \\
& =\frac{1}{(2 \pi)^{2}} \int_{\Im z=\eta} d z e^{\sqrt{-1}\left(z^{3} / 3+x z\right)}\left\{\int_{\Im w<c-\eta} d w-\int_{\Im w>c-\eta} d w\right\} \frac{e^{\sqrt{-1}\left(w^{3} / 3+y w\right)}}{c+\sqrt{-1}(z+w)}
\end{aligned}
$$

It is equal to the integral

$$
\frac{1}{2 \pi} \int_{\Im z=\eta} d z e^{\sqrt{-1}\left(z^{3} / 3+x z\right)} \frac{1}{2 \pi \sqrt{-1}} \oint_{C} d w \frac{e^{\sqrt{-1}\left(w^{3} / 3+\sqrt{-1} y w\right)}}{w-(\sqrt{-1} c-z)},
$$

where $C$ is a closed contour on $\mathbb{C}$ encircling a pole at $w=\sqrt{-1} c-z$ once in the positive direction. By performing the Cauchy integral, we have

$$
\begin{aligned}
I & =\frac{1}{2 \pi} \int_{\Im z=\eta} d z e^{\sqrt{-1}\left(z^{3} / 3+x z\right)} e^{\sqrt{-1}(\sqrt{-1} c-z)^{3} / 3-y(c+\sqrt{-1} z)} \\
& =\frac{1}{2 \pi} e^{c^{3} / 3-c y} \int_{\Im z=\eta} d z e^{-c z^{2}+\sqrt{-1}\left(x-y+c^{2}\right) z} .
\end{aligned}
$$

By performing a Gaussian integral, we have (3.4). Since

$$
-\frac{1}{4 c}(x-y)^{2}-\frac{c}{2}(x+y)+\frac{c^{3}}{12}=-\frac{1}{4 c}\left\{y-\left(x-c^{2}\right)\right\}^{2}-c x+\frac{c^{3}}{3},
$$

the Gaussian integration of (3.4) with respect to $y$ gives (3.5).

By setting $c=t / 2>0$ in (3.5) and $c=(t-s) / 2>0$ in (3.4), respectively, we obtain the equalities

$$
\begin{aligned}
& \int_{\mathbb{R}} d y \int_{\mathbb{R}} d u e^{u t} \operatorname{Ai}(u+x) \operatorname{Ai}(u+y)=g(t, x), \\
& \int_{\mathbb{R}} d u e^{u(t-s) / 2} \operatorname{Ai}(u+x) \operatorname{Ai}(u+y)=\frac{g(t, y)}{g(s, x)} q(s, t, y-x)
\end{aligned}
$$


with (1.28) and (1.30). Thus we have defined $g(t, x)$ for any $t \in \mathbb{R}$ by (1.28) and $p_{\mathrm{Ai}}(s, x ; t, y)$ for any $s, t \in \mathbb{R}, s \neq t$ by (1.31). As special cases, we have

$$
\begin{aligned}
p_{\mathrm{Ai}}(t, y \mid x) & =g(t, y) q(0, t, y-x), \\
p_{\mathrm{Ai}}(-t, y \mid x) & =\frac{1}{g(t, x)} q(t, 0, y-x), \quad t>0, x, y, \in \mathbb{R} .
\end{aligned}
$$

If we take the $c \rightarrow 0$ limit in (3.5), we obtain

$$
\int_{\mathbb{R}} d \xi \int_{\mathbb{R}} d x \operatorname{Ai}(\xi-x) \operatorname{Ai}\left(\xi^{\prime}-x\right)=1
$$

The expression (3.4) and the above result implies the orthonormality of the Airy function in the sense;

$$
\left(\int_{\mathbb{R}} d u \operatorname{Ai}(u+x) \operatorname{Ai}(u+y)\right) d y=\delta_{x}(d y)
$$

The Airy transform $f(x) \mapsto \varphi(\xi)$ is then defined by

$$
\varphi(\xi)=\int_{\mathbb{R}} d x f(x) \operatorname{Ai}(\xi+x),
$$

and the inverse transform is given by $f(x)=\int_{\mathbb{R}} d \xi \varphi(\xi) \mathrm{Ai}(\xi+x)$. Now a parameter $c \in \mathbb{C}$ is introduced and the family of functions are defined as $\left\{w_{c}(x)=\operatorname{Ai}(x / c) /|c|\right\}$. The Airy transform (3.9) is then generalized as

$$
\varphi_{c}(\xi)=\int_{\mathbb{R}} d \xi f(x) w_{c}(\xi+x)=\frac{1}{|c|} \int_{\mathbb{R}} d x f(x) \mathrm{Ai}\left(\frac{\xi+x}{c}\right) .
$$

Lemma 3.2 The Airy transform with c of the normalized Gaussian function $f(x)=$ $e^{-x^{2}} / \sqrt{\pi}$ is given by $\varphi_{c}(\xi)=|c|^{-1} e^{\left\{\xi+1 /\left(24 c^{3}\right)\right\} /\left(4 c^{3}\right)} \mathrm{Ai}\left(\xi / c+1 /\left(16 c^{4}\right)\right)$. That is,

$$
\int_{\mathbb{R}} d x \frac{1}{\sqrt{\pi}} e^{-x^{2}} \operatorname{Ai}\left(\frac{\xi+x}{c}\right)=\exp \left\{\frac{1}{4 c^{3}}\left(\xi+\frac{1}{24 c^{3}}\right)\right\} \operatorname{Ai}\left(\frac{\xi}{c}+\frac{1}{16 c^{4}}\right) .
$$

Proof. By the definition of the Airy function (1.4),

$$
\begin{aligned}
I & =\int_{\mathbb{R}} d x \frac{1}{\sqrt{\pi}} e^{-x^{2}} \operatorname{Ai}\left(\frac{\xi+x}{c}\right) \\
& =\int_{\mathbb{R}} d x \frac{1}{\sqrt{\pi}} e^{-x^{2}} \frac{1}{2 \pi} \int_{\mathbb{R}} d k e^{\sqrt{-1}\left\{k^{3} / 3+(\xi+x) k / c\right\}} \\
& =\frac{1}{2 \pi} \int_{\mathbb{R}} d k e^{\sqrt{-1} k^{3} / 3+\sqrt{-1} \xi k / c} \frac{1}{\sqrt{\pi}} \int_{\mathbb{R}} d x \exp \left(-x^{2}-\sqrt{-1} \frac{k}{c} x\right) .
\end{aligned}
$$


By performing the Gaussian integral we have

$$
I=\frac{1}{2 \pi} \int_{\mathbb{R}} d k \exp \left(\sqrt{-1} \frac{k^{3}}{3}+\sqrt{-1} \frac{\xi k}{c}-\frac{k^{2}}{4 c^{2}}\right) .
$$

By completing a cube, we find the equality

$$
\begin{aligned}
& \sqrt{-1} \frac{k^{3}}{3}+\sqrt{-1} \frac{\xi k}{c}-\frac{k^{2}}{4 c^{2}}=\sqrt{-1} \frac{1}{3}\left(k+\sqrt{-1} \frac{1}{4 c^{2}}\right)^{3} \\
& \quad+\sqrt{-1}\left(\frac{\xi}{c}+\frac{1}{16 c^{4}}\right)\left(k+\sqrt{-1} \frac{1}{4 c^{2}}\right)+\frac{1}{4 c^{3}}\left(\xi+\frac{1}{24 c^{3}}\right) .
\end{aligned}
$$

By using the definition of the Airy function (1.4), (3.10) is obtained.

For $t>0, y, u \in \mathbb{R}$ we will obtain the equality

$$
\int_{\sqrt{-1} \mathbb{R}} \frac{d z}{\sqrt{-1}} \operatorname{Ai}(u+z) q(t, 0, z-y)=\int_{\mathbb{R}} d x \frac{1}{\sqrt{\pi}} e^{-x^{2}} \operatorname{Ai}\left(\sqrt{-2 t} x+u+y-\frac{t^{2}}{4}\right)
$$

by changing the integral variable as $z \mapsto x=\left(z-y+t^{2} / 4\right) / \sqrt{-2 t}$. If we set $\xi=\left(u+y-t^{2} / 4\right) / \sqrt{-2 t}$ and $c=1 / \sqrt{-2 t}$, the RHS is identified with the LHS of (3.10). Since

$$
\frac{\xi}{c}+\frac{1}{16 c^{4}}=u+y, \quad \frac{1}{4 c^{3}}\left(\xi+\frac{1}{24 c^{3}}\right)=\left(-\frac{t y}{2}+\frac{t^{3}}{24}\right)-\frac{u t}{2},
$$

(3.10) of Lemma 3.2 with $(1.28)$ gives

$$
\int_{\sqrt{-1} \mathbb{R}} \frac{d z}{\sqrt{-1}} \operatorname{Ai}(u+z) q(t, 0, z-y)=g(t, y) e^{-u t / 2} \operatorname{Ai}(u+y), \quad t>0, y, u \in \mathbb{R} .
$$

Combination with (3.7) gives

$$
\int_{\sqrt{-1} \mathbb{R}} \frac{d z}{\sqrt{-1}} \operatorname{Ai}(u+z) p_{\mathrm{Ai}}(-t, z \mid y)=e^{-u t / 2} \operatorname{Ai}(u+y), \quad t>0, y, u \in \mathbb{R}
$$

\subsection{Fourier-Airy series}

Let us consider the integral

$$
I_{\ell \ell^{\prime}}=\int_{0}^{\infty} d x \operatorname{Ai}\left(x+a_{\ell}\right) \operatorname{Ai}\left(x+a_{\ell^{\prime}}\right), \quad a_{\ell}, a_{\ell^{\prime}} \in \mathcal{A} .
$$

In the case $\ell \neq \ell^{\prime}$, the formula (3.2) gives

$$
I_{\ell \ell^{\prime}}=\frac{\operatorname{Ai}^{\prime}\left(a_{\ell}\right) \operatorname{Ai}\left(a_{\ell^{\prime}}\right)-\operatorname{Ai}\left(a_{\ell}\right) \operatorname{Ai}^{\prime}\left(a_{\ell^{\prime}}\right)}{a_{\ell}-a_{\ell^{\prime}}}=0,
$$


whereas if $\ell=\ell^{\prime}$, the formula (3.1) gives $I_{\ell \ell}=\left(\operatorname{Ai}^{\prime}\left(a_{\ell}\right)\right)^{2}$. Therefore the functions

$$
\left\{\frac{\operatorname{Ai}\left(x+a_{\ell}\right)}{\operatorname{Ai}^{\prime}\left(a_{\ell}\right)}, \ell \in \mathbb{N}\right\}
$$

form an orthogonal basis for $f \in L^{2}(0, \infty)$ (see Sect. 4.12 in [27]). The completeness of $(3.12)$ is also established :

$$
\sum_{\ell \in \mathbb{N}} \frac{\operatorname{Ai}\left(x+a_{\ell}\right) \operatorname{Ai}\left(y+a_{\ell}\right)}{\left(\operatorname{Ai}^{\prime}\left(a_{\ell}\right)\right)^{2}} d y=\delta_{x}(d y), \quad x, y \in(0, \infty) .
$$

Then for any $f \in L^{2}(0, \infty)$, we can write the expression

$$
f(x)=\sum_{\ell \in \mathbb{N}} c_{\ell} \frac{\operatorname{Ai}\left(x+a_{\ell}\right)}{\operatorname{Ai}^{\prime}\left(a_{\ell}\right)}, \quad x \in[0, \infty),
$$

and call it the Fourier-Airy series expansion. The coefficients $c_{\ell}$ of this expansion are determined by $c_{\ell}=\left\{\int_{0}^{\infty} d x f(x) \operatorname{Ai}\left(x+a_{\ell}\right)\right\} / \operatorname{Ai}^{\prime}\left(a_{\ell}\right)$.

\section{Proof of Results}

\subsection{Proof of Proposition 2.4}

With (1.13) we put

$$
\widehat{g}(s, x)=\exp \left\{-D_{\mathcal{A}_{N}}\left(\frac{D_{\mathcal{A}_{N}} s}{2}+\frac{s^{2}}{4}-x\right)\right\}, s, x \in \mathbb{R} .
$$

By the definition (1.30) of $q$, for $s>0, x, x^{\prime} \in \mathbb{R}$, we have

$$
\begin{aligned}
\frac{p_{\sin }\left(s,\left(x-D_{\mathcal{A}_{N}} s-s^{2} / 4\right)-x^{\prime}\right)}{q\left(0, s, x-x^{\prime}\right)} \\
=\exp \left[-\frac{1}{2 s}\left\{\left(x-D_{\mathcal{A}_{N}} s-\frac{s^{2}}{4}-x^{\prime}\right)^{2}-\left(x-\frac{s^{2}}{4}-x^{\prime}\right)^{2}\right\}\right] \\
=\exp \left[-D_{\mathcal{A}_{N}} s\left(\frac{D_{\mathcal{A}_{N}} s}{2}+\frac{s^{2}}{4}-x+x^{\prime}\right)\right] \\
=\widehat{g}(s, x) e^{-D_{\mathcal{A}_{N}} x^{\prime}},
\end{aligned}
$$

and for $t>0, y, y^{\prime} \in \mathbb{R}$, we have

$$
\frac{p_{\sin }\left(-t, y^{\prime}-\left(y-D_{\mathcal{A}_{N}} t-t^{2} / 4\right)\right)}{q\left(t, 0, y^{\prime}-y\right)}=\frac{1}{\widehat{g}(t, y)} e^{D_{\mathcal{A}_{N}} y^{\prime}} .
$$


Similarly, we have

$$
\begin{aligned}
& \frac{p_{\sin }\left(s-t,\left(x-D_{\mathcal{A}_{N}} s-s^{2} / 4\right)-\left(y-D_{\mathcal{A}_{N}} t-t^{2} / 4\right)\right)}{q(t, s, y-x)} \\
& =\exp \left[-\frac{1}{2(s-t)}\left\{\left(x-y-D_{\mathcal{A}_{N}}(s-t)-\frac{s^{2}-t^{2}}{4}\right)^{2}\right.\right. \\
& \left.\left.\quad-\left(\left(x-\frac{s^{2}}{4}\right)-\left(y-\frac{t^{2}}{4}\right)\right)^{2}\right\}\right] \\
& =\exp \left[-D_{\mathcal{A}_{N}}\left\{\frac{D_{c A_{N}}}{2}(s-t)+\frac{s^{2}-t^{2}}{4}-(x-y)\right\}\right]=\frac{\widehat{g}(s, x)}{\widehat{g}(t, y)} .
\end{aligned}
$$

Then we have

$$
\begin{aligned}
& \mathbb{K}^{\xi^{N}}\left(s, x-D_{\mathcal{A}_{N}} s-\frac{s^{2}}{4} ; t, y-D_{\mathcal{A}_{N}} t-\frac{t^{2}}{4}\right) \\
& =\frac{\widehat{g}(s, x)}{\widehat{g}(t, y)}\left[\int_{\mathbb{R}} \xi^{N}\left(d x^{\prime}\right) \int_{\sqrt{-1} \mathbb{R}} \frac{d y^{\prime}}{\sqrt{-1}}\right. \\
& \times q\left(0, s, x-x^{\prime}\right) e^{-D_{\mathcal{A}_{N}} x^{\prime}} \Phi_{0}\left(\xi^{N}, x^{\prime}, y^{\prime}\right) e^{D_{\mathcal{A}_{N}} y^{\prime}} q\left(t, 0, y^{\prime}-y\right) \\
& -\mathbf{1}(s>t) q(t, s, x-y)] .
\end{aligned}
$$

The identity (2.12) implies

$$
e^{-D_{\mathcal{A}_{N}} x^{\prime}} \Phi_{0}\left(\xi^{N}, x^{\prime}, y^{\prime}\right) e^{D_{\mathcal{A}_{N}} y^{\prime}}=\Phi_{\mathcal{A}}\left(\xi^{N}, x^{\prime}, y^{\prime}\right) .
$$

By the gauge invariance of determinantal processes (Lemma 2.1), the proof is completed.

\subsection{Proof of Theorem 2.5}

First we prepare some lemmas for proving Theorem 2.5.

Lemma 4.1 Let $\alpha \in(1,2)$ and $\delta>\alpha-1$. Suppose that $M_{\alpha}(\xi)<\infty$ and put $L_{0}=L_{0}(\alpha, \delta, \xi)=\left(2 M_{\alpha}(\xi)\right)^{\alpha /(\delta-\alpha+1)}$. Then

$$
M_{1}(\xi, L) \leq L^{\delta}, \quad L \geq L_{0} .
$$

Since this lemma was proved as Lemma 4.3 in [13], here we omit the proof.

Lemma 4.2 If $\xi$ satisfies (C.2) (i) and (ii), for any $\theta \in(\alpha \vee(2-\beta), 2)$ there exists $C=C\left(C_{1}, C_{2}, \theta\right)>0$ such that

$$
\left|\int_{\{0, a\}^{\mathrm{c}}}\left(\frac{1}{x}-\frac{1}{x-a}\right) \xi(d x)\right| \leq C|a \vee 1|^{\theta-1}, \quad a \in \operatorname{supp}\left(\xi-\delta_{0}\right) .
$$


Proof. We divide $\{0, a\}^{\mathrm{c}}$ into three sets $A_{1}=\left\{x \in\{0, a\}^{\mathrm{c}}:|x| \leq|a| / 2\right\}, A_{2}=\{x \in$ $\left.\{0, a\}^{\mathrm{c}}:|a| / 2<|x| \leq 2|a|\right\}$, and $A_{3}=\left\{x \in\{0, a\}^{\mathrm{c}}: 2|a|<|x|\right\}$, and put

$$
I_{j}=\int_{A_{j}}\left|\frac{1}{x}-\frac{1}{x-a}\right| \xi(d x) \quad j=1,2,3 .
$$

When $x \in A_{1},\left|x^{2}-a^{2}\right| \geq 3 a^{2} / 4$ and $|x+a| \leq 3|a| / 2$, and then

$$
I_{1}=\int_{A_{1}} \frac{|a||x+a|}{|x|\left|x^{2}-a^{2}\right|} \xi(d x) \leq 2 M_{1}\left(\xi, \frac{|a|}{2}\right) .
$$

By Lemma 4.1 for any $\delta>\alpha-1$, we can take $C>0$ such that

$$
I_{1} \leq C|a \vee 1|^{\delta}
$$

When $x \in A_{2},|x+a| \leq 3|a|$ and $|a| /|x| \leq 2$, and then

$$
I_{2}=\int_{A_{2}} \frac{|a||x+a|}{|x|\left|x^{2}-a^{2}\right|} \xi(d x) \leq 6|a| M_{1}\left(\tau_{-a^{2}} \xi^{\langle 2\rangle}\right) .
$$

From the condition (C.2) (ii)

$$
I_{2} \leq 6 C_{2}|a \vee 1|^{1-\beta}
$$

When $x \in A_{3},|x-a|>|x| / 2$, and then

$$
I_{3}=\int_{A_{3}} \frac{|a|}{|x||x-a|} \xi(d x) \leq 2^{\alpha-1}|a|^{\alpha-1} M_{\alpha}(\xi)^{\alpha} .
$$

From the condition (C.2) (i)

$$
I_{3} \leq 2^{\alpha-1} C_{1}|a \vee 1|^{\alpha-1}
$$

Combining the estimates (4.2), (4.3) and (4.4), we have (4.1).

Lemma 4.3 (i) If $\xi$ satisfies the conditions (C.2) (i) and (ii), for any $\theta \in(\alpha \vee$ $(2-\beta), 2)$ there exists $C=C\left(C_{1}, C_{2}, \theta\right)>0$ such that

$$
\left|\Phi_{1}(\xi, a, \sqrt{-1} y)\right| \leq \exp \left[C\left\{\left(|y|^{\theta} \vee 1\right)+\left(|a|^{\theta} \vee 1\right)\right\}\right]
$$

for $y \in \mathbb{R}$ and $a \in \operatorname{supp} \xi$.

(ii) If $\xi$ satisfies the conditions (C.1), (C.2) (i) and (ii), for any $\theta \in(\alpha \vee(2-\beta), 2)$ there exists $C=C\left(C_{0}, C_{1}, C_{2}, \theta\right)>0$ such that

$$
\left|\Phi_{\mathcal{A}}(\xi, a, \sqrt{-1} y)\right| \leq \exp \left[C\left\{\left(|y|^{\theta} \vee 1\right)+\left(|a|^{\theta} \vee 1\right)\right\}\right]
$$

for $y \in \mathbb{R}$ and $a \in \operatorname{supp} \xi$. 
Proof. We prove (i) of this lemma. From the condition (C.1) and the relation (2.12), (ii) is easily derived from (i). We first consider the case that $a=0 \in \operatorname{supp} \xi$. Remind that

$$
\Phi_{1}(\xi, 0, z)=\Pi_{1}(\xi, z)=\exp \left[\int_{\{0\}^{\mathrm{c}}}\left\{\log \left(1-\frac{z}{x}\right)+\frac{z}{x}\right\} \xi(d x)\right] .
$$

When $2|z|<|x|$, by using the expansion

$$
\log \left(1-\frac{z}{x}\right)=-\sum_{k \in \mathbb{N}} \frac{1}{k}\left(\frac{z}{x}\right)^{k}
$$

we have

$$
\left|\log \left(1-\frac{z}{x}\right)+\frac{z}{x}\right| \leq\left|\frac{z}{x}\right|^{2}
$$

Then

$$
\left|\Pi_{1}\left(\xi \cap[-2|z|, 2|z|]^{\mathrm{c}}, z\right)\right| \leq \exp \left\{|z|^{2} \int_{|x|>2|z|} \frac{1}{x^{2}} \xi(d x)\right\} \leq \exp \left\{|z|^{\alpha} M_{\alpha}(\xi)^{\alpha}\right\} .
$$

On the other hand $|1-z / x| \leq e^{|z| /|x|}$. Then

$$
\left|\Pi_{1}(\xi \cap[-2|z|, 2|z|], z)\right| \leq \exp \left\{2|z| \int_{|x| \leq 2|z|} \frac{1}{|x|} \xi(d x)\right\}=\exp \left\{2|z| M_{1}(\xi, 2|z|)\right\} .
$$

From (4.7) and (4.8), with the condition (C.2) (i) and Lemma 4.1, we see that for any $\theta \in(\alpha \vee(2-\beta), 2)$ there exists $C=C\left(C_{1}, \theta\right)>0$ such that

$$
\left|\Phi_{1}(\xi, 0, z)\right| \leq \exp \left[C\left\{\left(|z|^{\theta} \vee 1\right)\right\}\right]
$$

for $z \in \mathbb{C}$ and $a \in \operatorname{supp} \xi$.

Next we consider the case that $a \in \operatorname{supp} \xi$ and $a \neq 0$. By the conditions (C.1) and (C.2) the equality (2.16) is valid. By (4.9)

$$
\left|\Pi_{1}(\xi, z) \Pi_{1}\left(\xi \cap\{-a\}^{\mathrm{c}},-a\right)\right| \leq \exp \left[C\left\{\left(|z|^{\theta} \vee 1\right)+\left(|a|^{\theta} \vee 1\right)\right\}\right] .
$$

By the condition (C.2) (ii)

$$
\left|\Phi_{0}\left(\xi^{\langle 2\rangle} \cap\{0\}^{\mathrm{c}}, a^{2}, 0\right)\right| \leq \exp \left\{|a|^{2} M_{1}\left(\tau_{-a^{2}} \xi^{\langle 2\rangle}\right)\right\} \leq \exp \left\{C_{2}(|a| \vee 1)^{2-\beta}\right\},
$$

and $\left|(\sqrt{-1} y / a)^{\xi(\{0\})} a /(a-\sqrt{-1} y)\right| \leq 1$. Now we evaluate $S(\xi, a, z)$.

$$
\begin{aligned}
& S(\xi, a, z)=\int_{\{0, a\}^{\mathrm{c}}}\left(\frac{z-a}{x-a}-\frac{z-a}{x}\right) \xi(d x) \\
& \quad+\frac{(z-a)}{-a} \xi(\{0\})-\frac{z}{a}+\frac{a}{a}-\frac{a}{-a} \xi(\{-a\}) \\
& =(z-a) \int_{\{0, a\}^{\mathrm{c}}}\left(\frac{1}{x-a}-\frac{1}{x}\right) \xi(d x)-\frac{(1+\xi(\{0\})) z}{a}+1+\xi(\{0\})+\xi(\{-a\}) .
\end{aligned}
$$


From Lemma 4.2 and the fact $1 / a^{2} \leq C_{2}$ and then $|2 z / a| \leq 2 \sqrt{C_{2}}|z|$, we have

$$
|S(\xi, a, z)| \leq C|z-a||a \vee 1|^{\theta-1}+2 \sqrt{C_{2}}|z|+3 \leq C^{\prime}\left\{\left(|y|^{\theta} \vee 1\right)+\left(|a|^{\theta} \vee 1\right)\right\}
$$

for some $C^{\prime}>0$. This completes the proof.

Proof of Theorem 2.5. Note that $\xi \cap[-L, L], L>0$ and $\xi$ satisfy (C.1) and (C.2) with the same constants $C_{0}, C_{1}, C_{2}$ and indices $\alpha, \beta$. By virtue of Lemma 4.3 (ii) we see that there exists $C>0$ such that

$$
\left|\Phi_{\mathcal{A}}(\xi \cap[-L, L], a, \sqrt{-1} y)\right| \leq \exp \left[C\left\{(|y| \vee 1)^{\theta}+(|a| \vee 1)^{\theta}\right\}\right],
$$

$\forall L>0, \forall a \in \operatorname{supp} \xi, \forall y \in \mathbb{R}$. Since for any $y \in \mathbb{R}$

$$
\Phi_{\mathcal{A}}(\xi \cap[-L, L], a, \sqrt{-1} y) \rightarrow \Phi_{\mathcal{A}}(\xi, a, \sqrt{-1} y), \quad L \rightarrow \infty,
$$

we can apply Lebesgue's convergence theorem to (2.23) and obtain

$$
\lim _{L \rightarrow \infty} \mathbb{K}_{\mathcal{A}}^{\xi \cap[-L, L]}(s, x ; t, y)=\mathbb{K}_{\mathcal{A}}^{\xi}(s, x ; t, y) .
$$

Since for any $(s, t) \in(0, \infty)^{2}$ and any finite interval $I \subset \mathbb{R}$

$$
\sup _{x, y \in I}\left|\mathbb{K}_{\mathcal{A}}^{\xi \cap[-L, L]}(s, x ; t, y)\right|<\infty
$$

we can obtain the convergence of generating functions for multitime correlation functions (2.1) $; \mathcal{G}^{\xi \cap[-L, L]}[\chi] \rightarrow \mathcal{G}^{\xi}[\chi]$ as $L \rightarrow \infty$. It implies $\mathbb{P}_{\mathcal{A}}^{\xi \cap[-L, L]} \rightarrow \mathbb{P}_{\mathcal{A}}^{\xi}$ as $L \rightarrow \infty$ in the sense of finite dimensional distributions. Then the proof is completed.

\subsection{Proof of Theorem 2.6}

(i) It is clear that $\xi_{\mathcal{A}}$ is an element of $\mathfrak{X}_{\mathcal{A}}^{0}$. (See the item (1) of Sect. 2.4.) Then by Theorem $2.5\left(\Xi_{\mathcal{A}}(t), \mathbb{P}_{\mathcal{A}}^{\xi_{\mathcal{A}}^{N}}\right) \rightarrow\left(\Xi_{\mathcal{A}}(t), \mathbb{P}_{\mathcal{A}}^{\xi_{\mathcal{A}}}\right)$ as $N \rightarrow \infty$ in the sense of finite dimensional distributions, where $\left(\Xi_{\mathcal{A}}(t), \mathbb{P}_{\mathcal{A}}^{\xi_{\mathcal{A}}}\right)$ is the determinantal with the correlation kernel

$$
\begin{gathered}
\mathbb{K}_{\mathcal{A}}^{\xi_{\mathcal{A}}}(s, x ; t, y)=\int_{\mathbb{R}} \xi_{\mathcal{A}}(d a) \int_{\sqrt{-1} \mathbb{R}} \frac{d z}{\sqrt{-1}} q(0, s, x-a) \Phi_{\mathcal{A}}\left(\xi_{\mathcal{A}}, a, z\right) q(t, 0, z-y) \\
-\mathbf{1}(s>t) q(t, s, x-y) .
\end{gathered}
$$

Using the equalities (3.6) and (3.7) and the definition (1.31), we have

$$
\begin{aligned}
\mathbb{K}_{\mathcal{A}}^{\xi_{\mathcal{A}}}(s, x ; t, y)= & \int_{\mathbb{R}} \xi_{\mathcal{A}}(d a) \int_{\sqrt{-1} \mathbb{R}} \frac{d z}{\sqrt{-1}} \frac{p_{\mathrm{Ai}}(s, x \mid a)}{g(s, x)} \frac{1}{z-a} \frac{\operatorname{Ai}(z)}{\operatorname{Ai}^{\prime}(a)} g(t, y) p_{\mathrm{Ai}}(-t, z \mid y) \\
& \quad-\mathbf{1}(s>t) \frac{g(t, y)}{g(s, x)} p_{\mathrm{Ai}}(s-t, x \mid y) \\
= & \frac{g(t, y)}{g(s, x)} \mathbb{K}_{\mathrm{Ai}}(s, x ; t, y)
\end{aligned}
$$


with (2.26), where we have used (2.18) of Lemma 2.3. By the gauge invariance, Lemma 2.1, $\left(\Xi_{\mathcal{A}}(t), \mathbb{P}_{\mathcal{A}}^{\xi_{\mathcal{A}}}\right)=\left(\Xi_{\mathcal{A}}(t), \mathbb{P}_{\mathrm{Ai}}\right)$ in the sense of finite dimensional distributions.

(ii) If we use the expression (3.3), (2.26) becomes

$$
\begin{aligned}
\mathbb{K}_{\mathrm{Ai}}(s, x ; t, y)= & \sum_{a \in \mathrm{Ai}^{-1}(0)} \int_{\sqrt{-1} \mathbb{R}} \frac{d z}{\sqrt{-1}} p_{\mathrm{Ai}}(s, x \mid a) \\
& \times \frac{1}{\left(\operatorname{Ai}^{\prime}(a)\right)^{2}} \int_{0}^{\infty} d u \operatorname{Ai}(u+z) \operatorname{Ai}(u+a) p_{\mathrm{Ai}}(-t, z \mid y) \\
& -\mathbf{1}(s>t) p_{\mathrm{Ai}}(s-t, x \mid y) .
\end{aligned}
$$

By (3.11), the first term of the RHS equals

$$
\sum_{a \in \mathrm{Ai}^{-1}(0)} p_{\mathrm{Ai}}(s, x \mid a) \frac{1}{\left(\operatorname{Ai}^{\prime}(a)\right)^{2}} \int_{0}^{\infty} d u e^{-u t / 2} \operatorname{Ai}(u+y) \operatorname{Ai}(u+a) .
$$

Since $s>0$, we can use the expression (1.27) for $p_{\mathrm{Ai}}(s, x \mid a)$ and the above is written as

$$
\int_{0}^{\infty} d u \int_{\mathbb{R}} d w e^{-u t / 2+w s / 2} \operatorname{Ai}(u+y) \operatorname{Ai}(w+x) \sum_{\ell \in \mathbb{N}} \frac{\operatorname{Ai}\left(u+a_{\ell}\right) \operatorname{Ai}\left(w+a_{\ell}\right)}{\left(\operatorname{Ai}^{\prime}\left(a_{\ell}\right)\right)^{2}} .
$$

From the completeness (3.13), the above gives

$$
\mathbb{K}_{\mathrm{Ai}}(s, x ; t, y)=\mathbf{K}_{\mathrm{Ai}}(t-s, y \mid x)+R(s, x ; t, y)
$$

with the extended Airy kernel $\mathbf{K}_{\mathrm{Ai}}$ given by (1.37) and

$$
\begin{gathered}
R(s, x ; t, y)=\int_{0}^{\infty} d u \int_{-\infty}^{0} d w e^{-u t / 2+w s / 2} \operatorname{Ai}(u+y) \operatorname{Ai}(w+x) \\
\times \sum_{\ell \in \mathbb{N}} \frac{\operatorname{Ai}\left(u+a_{\ell}\right) \operatorname{Ai}\left(w+a_{\ell}\right)}{\left(\operatorname{Ai}^{\prime}\left(a_{\ell}\right)\right)^{2}}
\end{gathered}
$$

Since for any fixed $s, t>0 \lim _{\theta \rightarrow \infty}|R(s+\theta, x ; t+\theta, y)| \rightarrow 0$ uniformly on any compact subset of $\mathbb{R}^{2}$, (1.36) holds in the same sense. Hence we obtain (2.27). This completes the proof.

Acknowledgments. M.K. is supported in part by the Grant-in-Aid for Scientific Research (C) (No.21540397) of Japan Society for the Promotion of Science. H.T. is supported in part by the Grant-in-Aid for Scientific Research (KIBAN-C, No.19540114) of Japan Society for the Promotion of Science. 


\section{References}

[1] Abramowitz, M., Stegun, I. : Handbook of Mathematical Functions. Dover, New York (1965)

[2] Adler, M., van Moerbeke, P. : The spectrum of coupled random matrices. Ann. Math. 149, 921-976 (1999)

[3] Adler, M., van Moerbeke, P. : PDF's for the joint distributions of the Dyson, Airy and Sine processes. Ann. Probab. 33, 1326-1361 (2005)

[4] Dyson, F. J. : A Brownian-motion model for the eigenvalues of a random matrix. J. Math. Phys. 3, 1191-1198 (1962)

[5] Dyson, F. J. : The three fold way. Algebraic structure of symmetry groups and ensembles in quantum mechanics. J. Math. Phys. 3, 1199-1215 (1962)

[6] Ferrari, P. L., Spohn, H. : Constrained Brownian motion : Fluctuations away from circular and parabolic barriers. Ann. Probab. 33, 1302-1325 (2005)

[7] Flajolet, P., Louchard, G. : Analytic variations on the Airy distribution. Algorithmica 31, 361-377 (2001)

[8] Forrester, P. J. : The spectrum edge of random matrix ensembles. Nucl. Phys. B 402, 709-728 (1993)

[9] Forrester, P. J., Nagao, T., Honner, G. : Correlations for the orthogonal-unitary and symplectic-unitary transitions at the hard and soft edges. Nucl. Phys. B 553(PM), 601-643 (1999)

[10] Grabiner, D. J.: Brownian motion in a Weyl chamber, non-colliding particles, and random matrices. Ann. Inst. Henri Poincaré, Probab. Stat. 35, 177-204 (1999)

[11] Johansson, K. : Discrete polynuclear growth and determinantal processes. Commun. Math. Phys. 242, 277-329 (2003)

[12] Katori, M., Tanemura, H.: Noncolliding Brownian motion and determinantal processes. J. Stat. Phys. 129, 1233-1277 (2007)

[13] Katori, M., Tanemura, H.: Non-equilibrium dynamics of Dyson's model with an infinite number of particles. Commun. Math. Phys. doi:10.1007/s00220-009-0912-3. arXiv:0812.4108[math.PR]

[14] Katori, M., Tanemura, H.: in preparation

[15] Katori, M., Nagao, T., Tanemura, H. : Infinite systems of non-colliding Brownian particles. In: Stochastic Analysis on Large Scale Interacting Systems. Adv. Stud. in Pure Math., vol. 39, pp.283-306. Mathematical Society of Japan, Tokyo (2004). arXiv:math.PR/0301143

[16] Levin, B. Ya.: Lectures on Entire Functions. Translations of Mathematical Monographs, vol.150. Amer. Math. Soc., Providence (1996) 
[17] Mehta, M. L. : Random Matrices, 3rd edn. Elsevier, Amsterdam (2004)

[18] Nagao, T., Forrester, P. J. : Multilevel dynamical correlation functions for Dyson's Brownian motion model of random matrices. Phys. Lett. A247, 42-46 (1998)

[19] Nagao, T., Katori, M., H. Tanemura, H. : Dynamical correlations among vicious random walkers. Phys. Lett. A 307, 29-35 (2003)

[20] Osada, H. : Dirichlet form approach to infinite-dimensional Wiener processes with singular interactions. Commun. Math. Phys. 176, 117-131 (1996)

[21] Osada, H. : Interacting Brownian motions in infinite dimensions with logarithmic interaction potentials. arXiv:0902.3561 [math.PR]

[22] Prähofer, M., Spohn, H. : Scale invariance of the PNG droplet and the Airy process. J. Stat. Phys. 108, 1071-1106 (2002)

[23] Shirai, T., Takahashi, Y.: Random point fields associated with certain Fredholm determinants I: fermion, Poisson and boson point process. J. Funct. Anal. 205, 414$463(2003)$

[24] Soshnikov, A. : Determinantal random point fields. Russian Math. Surveys 55, 923$975(2000)$

[25] Spohn, H. : Interacting Brownian particles: A study of Dyson's model. In: Papanicolaou, G. (ed.) Hydrodynamic Behavior and Interacting Particle Systems, IMA Volumes in Mathematics and its Applications, vol. 9, pp.151-179. Springer, Berlin (1987)

[26] Spohn, H. : Large Scale Dynamics of Interacting Particles. Springer, Berlin (1991)

[27] Titchmarsh, E. C. : Eigenfunction Expansions Associated with Second-order Differential Equations. Part I, 2nd edn. Clarendon Press, Oxford (1962)

[28] Tracy, C. A., Widom, H. : Level-spacing distributions and the Airy kernel. Commun. Math. Phys. 159, 151-174 (1994)

[29] Tracy, C. A., Widom, H. : A system of differential equations for the Airy process. Elect. Commun. Probab. 8, 93-98 (2003)

[30] Vallée, O., Soares, M. : Airy Functions and Applications to Physics. Imperial College Press, London (2004) 